\title{
Development of a dynamic headspace solid-phase microextraction procedure coupled to GC-qMSD for evaluation the chemical profile in alcoholic beverages
}

\author{
F. Rodrigues, M. Caldeira, J.S. Câmara* \\ Centro de Química da Madeira, Departamento de Química, Universidade da Madeira, \\ Campus Universitário da Penteada, 9000-390 Funchal, Portugal
}

\section{A R T I C L E I N F O}

\section{Article history:}

Received 7 September 2007

Received in revised form

19 December 2007

Accepted 27 December 2007

Published on line 8 January 2008

\section{Keywords:}

Volatile and semi-volatile

compounds

Headspace solid-phase

microextraction

White wine

Beer

Whisky

GC-qMSD

\section{A B S T R A C T}

In the present study, a simple and sensitive methodology based on dynamic headspace solidphase microextraction (HS-SPME) followed by thermal desorption gas chromatography with quadrupole mass detection (GC-qMSD), was developed and optimized for the determination of volatile (VOCs) and semi-volatile (SVOCs) compounds from different alcoholic beverages: wine, beer and whisky. Key experimental factors influencing the equilibrium of the VOCs and SVOCs between the sample and the SPME fibre, as the type of fibre coating, extraction time and temperature, sample stirring and ionic strength, were optimized. The performance of five commercially available SPME fibres was evaluated and compared, namely polydimethylsiloxane (PDMS, $100 \mu \mathrm{m}$ ); polyacrylate (PA, $85 \mu \mathrm{m}$ ); polydimethylsiloxane/divinylbenzene (PDMS/DVB, $65 \mu \mathrm{m}$ ); carboxen ${ }^{\mathrm{TM}} /$ polydimethylsiloxane (CAR/PDMS, $75 \mu \mathrm{m}$ ) and the divinylbenzene/carboxen on polydimethylsiloxane (DVB/CAR/PDMS, 50/30 $\mu \mathrm{m}$ ) (StableFlex).

An objective comparison among different alcoholic beverages has been established in terms of qualitative and semi-quantitative differences on volatile and semi-volatile compounds. These compounds belong to several chemical families, including higher alcohols, ethyl esters, fatty acids, higher alcohol acetates, isoamyl esters, carbonyl compounds, furanic compounds, terpenoids, C13-norisoprenoids and volatile phenols. The optimized extraction conditions and GC-qMSD, lead to the successful identification of 44 compounds in white wines, 64 in beers and 104 in whiskys. Some of these compounds were found in all of the examined beverage samples.

The main components of the HS-SPME found in white wines were ethyl octanoate (46.9\%), ethyl decanoate $(30.3 \%)$, ethyl 9-decenoate $(10.7 \%)$, ethyl hexanoate $(3.1 \%)$, and isoamyl octanoate $(2.7 \%)$. As for beers, the major compounds were isoamyl alcohol (11.5\%), ethyl octanoate (9.1\%), isoamyl acetate (8.2\%), 2-ethyl-1-hexanol (5.9\%), and octanoic acid (5.5\%). Ethyl decanoate $(58.0 \%)$, ethyl octanoate $(15.1 \%)$, ethyl dodecanoate $(13.9 \%)$ followed by 3 methyl-1-butanol $(1.8 \%)$ and isoamyl acetate $(1.4 \%)$ were found to be the major VOCs in whisky samples.

() 2008 Elsevier B.V. All rights reserved.

\footnotetext{
* Corresponding author. Tel.: +351 291705112; fax: +351 291705149.

E-mail address: jsc@uma.pt (J.S. Câmara).
} 


\section{Introduction}

Alcoholic beverages are highly complex mixtures of compounds, which largely define its appearance, aroma, flavour and mouth-feel properties. Being a combination of taste and olfaction properties, these characteristics are the most important parameters responsible for the beverages character, quality and hence for consumer acceptance. Their volatile fraction can be composed by over than 800 different compounds [1,2] but only several tens of which can be odour-active [3] and must be considered for differentiation purposes. These compounds belong to several chemical classes, including higher alcohols, ethyl esters, fatty acids, higer alcohol acetates, isoamyl esters, carbonyl compounds, sulphur compounds, furanic compounds, monoterpenols, $\mathrm{C}_{13}$-norisoprenoids and volatile phenols. They present different polarities, volatilities, and moreover, are found in a wide range of concentrations from $\mathrm{ng} \mathrm{L}^{-1}$ to $\mathrm{mgL}^{-1}$. They are produced through metabolic pathways during ripening, harvest, post-harvest and storage or ageing stages. In contrast to some wines and whisky, beer aging is usually considered negative to flavour quality. Many of these compounds are common to different alcoholic beverages but differ analytically in terms of relative amount. The different substances may influence their aroma and flavour both individually and in a synergistic or antagonistic sense. Some VOCs are of great importance and may contribute greatly to the alcoholic beverage flavour, while others are important merely in building up the background flavour of the product. The qualitative and quantitative study of beverages is an important database for ensuring process continuity and product authenticity [4].

The main higher alcohols (also known as fusel alcohols) found in alcoholic beverages are the aliphatic alcohols, $n$ propanol, 2-methylpropan-1-ol, 3-methylbutan-1-ol and the aromatic alcohols $\beta$-phenylethanol and benzyl alcohol. These compounds might have both positive and negative impacts on aroma and flavour. Excessive concentrations of higher alcohols $\left(>300 \mathrm{mg} \mathrm{L}^{-1}\right)$ can result in a strong, pungent smell and taste, whereas optimal levels impart fruity characters. Hexan-1-ol, for example, is usually a minor constituent, but its herbaceous and greasy odours have been related to deleterious effects in beverages, although consumers can appreciate a small herbaceous perception in white wines [5].

Other important compounds of beverages aroma are the fatty acids. The shorter chain fatty acids, such as acetic (the most important, quantitatively), propanoic, butanoic and lactic, are by-products of fermentation. Relatively few fatty acids in alcoholic beverages are volatile enough to contribute to its flavour. Odorous acids are, for example, acetic acid (vinegar-like character), propanoic acid (goaty) and butanoic acid (spoiled butter). Fatty acids are mainly formed during fermentation process at an earlier stage than the respective ethyl esters [6]. The straight-chain C8-C10 acids are intermediates in the formation of $\mathrm{C} 16$ and $\mathrm{C} 18$ acids (structural components of cell membranes).

Other compounds that can be sensory important are the ethyl esters (fruity flavours). The ethyl esters of straight-chain fatty acids and acetates of higher alcohols are the domi- nating esters in wine, beer and whisky. Are products of an enzyme catalysed condensation reaction between acyl-CoA and a higher alcohol [7]. Basically, two factors are important for the rate of ester formation: the concentration of the two substrates, acyl-CoA and fusel alcohol, and the total activity of the enzymes involved in the formation and breakdown of the respective ester. Qualitatively, the most significant esters are ethyl acetate (fruity, solvent-like), isoamyl acetate (banana, pear-drops aromas), isobutyl acetate (banana, fruity aroma), ethyl hexanoate (green apple aroma), ethyl octanoate (fruity, soap aroma), ethyl decanoate (floral, soap aroma) and 2-phenylethyl acetate (honey, fruity, flowery aromas). The shorter chain fatty acids ethyl esters contribute more to alcoholic beverage aroma than the less volatile longer chain esters [8]. The presence of different esters can have a synergistic effect on the individual flavours, which means that esters can also affect wine, beer and whisky flavour well below their individual threshold concentrations [9]. Moreover, the fact that most esters are present in concentrations around the threshold value implies that minor changes in concentration may have dramatic effects on beverage flavour.

The analysis of VOCs and SVOCs that contribute to alcoholic beverages (beer, wine and whisky) aroma, has been carried out following different methodologies, such as, liquid-liquid extraction [10] simultaneous distillation-extraction (SDE) [11], supercritical fluid extraction (SFE) [12], solid-phase extraction (SPE) [13] and ultrasound extraction [14]. Most of these techniques have several disadvantages, including extensive equipment requirements, significant quantities of expensive and environmentally unfriendly solvents, multiple handling steps that increase error, and a need for concentration of the target analytes to achieve detectable levels. Nowadays, they are easier and more selective alternatives to these classical methods, which may overcome their disadvantages, such as solid-phase microextraction (SPME), developed by Lord and Pawliszyn $[15,16]$ and more recently stir bar sorptive extraction (SBSE) developed in the late 1990s by Baltussen et al. [17]. This technique uses a Twister ${ }^{\mathrm{TM}}$, a glass stir bar onto which is bonded a sorptive phase, often polydimethylsiloxane (PDMS), in quantities far in excess of those found on SPME fibres [18]. These methods show clear advantages compared with traditional techniques: (i) eliminates the use of (toxic) organic solvents; (ii) allows the quantification of a large number of molecules with low limits of detection and good linearity over a considerable dynamic range; (iii) integrates sampling, extraction and concentration into a single step; (iv) requires no or little manipulation/preparation of sample; (v) substantially shortness the time of analysis; and moreover (vi) are simple and faster techniques, and (vii) covers a wide range of sampling techniques, including field, in situ and air sampling. Generally accepted disadvantages are relatively lot-to-lot variations, sensitivity against organic solvents and the limited range of commercially available stationary phases.

Since the first SPME fibres became commercially available, it has been more and more used and the fields of application have been continuously growing, including a wide range of food analysis, namely the volatile composition of wines [18-22], beers [23,24], whiskys [25-27] and several kinds of fruits [28-31], clinical chemistry [32], environmental chemistry 
$[33,34]$ and pharmaceutical analysis $[35,36]$, with nowadays about 3000 research papers published.

In this study, a fast and sensitive method based on manual dynamic headspace SPME sampling coupled with gas chromatography-quadrupole mass spectrometry detection (HS-SPME-GC-qMSD) was developed for the qualitative and semi-quantitative analysis of the volatile composition of alcoholic beverages and the main experimental factors which influence the extraction efficiency were optimized. Five commercially available SPME fibres: $100 \mu \mathrm{m}$ polydimethylsiloxane, PDMS, apolar; $85 \mu \mathrm{m}$ polyacrylate, PA, polar; $65 \mu \mathrm{m}$ polydimethylsiloxane/divinylbenzene, PDMS/DVB; $75 \mu \mathrm{m}$ carboxen ${ }^{\mathrm{TM}} /$ polydimethylsiloxane, CAR/PDMS and $50 / 30 \mu \mathrm{m}$ divinylbenzene/carboxen on polydimethylsiloxane, DVB/CAR/PDMS (StableFlex) polar, were tested and their performance compared. Finally, the developed and optimized methodology was successfully used to isolate and identify the main constituents of the volatile fraction of some commercial alcoholic beverages-beers, white wines and whiskys.

\section{Experimental}

\subsection{Chemicals and materials}

All used reagents were analytical quality and all solvents were HPLC grade. Sodium chloride (99.5\%), was used to obtain the adequate ionic strength, was supplied by Merck (Darmstadt, Germany). Absolute ethanol (purity $\geq 99.8 \%$ ) was from Panreac (Barcelone, Spain). The pure reference compounds and the chemical standard used as internal standard, 3-octanol, were supplied by Sigma-Aldrich (Spain). The $\mathrm{C}_{8}-\mathrm{C}_{20} n$-alkane series was supplied by Sigma-Aldrich (Spain). Water was purified through a Milli-Q purification system (Millipore).

The SPME fibres, polydimethylsiloxane layer (PDMS, $100 \mu \mathrm{m})$; polyacrylate (PA, $85 \mu \mathrm{m})$; divinylbenzenecarboxen-polydimethylsiloxane (DVB/CAR/PDMS, 50/30 $\mu \mathrm{m}$ ) on a $1 \mathrm{~cm}$ StableFlex fibre; carboxen-polydimethylsiloxane (CAR/PDMS, $75 \mu \mathrm{m}$ ); polydimethylsiloxane/divinylbenzene (PDMS/DVB, $65 \mu \mathrm{m}$ ) and the SPME holder for manual sampling, were obtained from Supelco (Bellefonte, PA, USA). The coating of all fibres was $1 \mathrm{~cm}$ long. The fibres were conditioned before the first use according to the manufacturer's instructions by inserting them into the GC injector port ( $100 \mu \mathrm{m}$ PDMS $0.5 \mathrm{~h}$ at $250^{\circ} \mathrm{C} ; 85 \mu \mathrm{m}$ PA $2 \mathrm{~h}$ at $300^{\circ} \mathrm{C} ; 50 / 30 \mu \mathrm{m} \mathrm{DVB} / \mathrm{CAR} / \mathrm{PDMS} 1 \mathrm{~h}$ at $270{ }^{\circ} \mathrm{C} ; 65 \mu \mathrm{m}$ PDMS/DVB $0.5 \mathrm{~h}$ at $250^{\circ} \mathrm{C}$ and $75 \mu \mathrm{m}$ CAR/PDMS $1.5 \mathrm{~h}$ at $300^{\circ} \mathrm{C}$ ). Before the first daily analysis the fibres were conditioned for $20 \mathrm{~min}$. A blank test was performed to check possible carry-over. If not in use for more than $24 \mathrm{~h}$, then the fibres were cleaned for $20 \mathrm{~min}$ at their respective conditioning temperature.

\subsection{Beverage samples}

Five Portuguese white wines (Terras Madeirenses) of the 2005 vintage, originating from different grape varieties (Table 1), all of them belonging to the Portuguese Madeira Appellation "Região Demarcada da Madeira", were analysed following the proposed method. The wine samples were supplied by
Table 1 - Varietal composition of Terras madeirenses wine samples (bold character indicates the main variety)

\begin{tabular}{|c|c|c|}
\hline Wine samples & Varietal composition & Alcohol (\%) \\
\hline$W W 1^{a}$ & Verdelho, Arnsburger, Boal & 11.5 \\
\hline wW2 & Verdelho, Arnsburger & 12.5 \\
\hline ww3 & Malvazia & 12.5 \\
\hline WW4 & Arnsburger & 12.0 \\
\hline WW5 & Verdelho, & 12.5 \\
\hline
\end{tabular}

the Madeira Wine Institute, and were produced in Adega de São Vicente (Northern of Madeira Island) on industrial scale using traditional winemaking processes for white wines. Grapes from different varieties were crushed, destemmed, racked and pressed. The musts were fermented in stainless-steel containers, with spontaneous yeast. Alcoholic fermentation was carried out at $18-20^{\circ} \mathrm{C}$. The code of analysed wines and the varietal composition of the different wine samples are presented in Table 1. All samples were taken from bottled wines $(750 \mathrm{ml})$ ready for sale and were stored at $-28^{\circ} \mathrm{C}$ until analysis. WW1 Vitis vinifera $\mathrm{L}$. white wine $(11.5 \%$, (v/v)), made with Verdelho, Arnsburger and Boal grapes, was selected for the HS-SPME method optimization.

Beer samples were purchased from the retail market and included beer "B1" 100\% malt, beers "B2" and "B3" 100\% malt (same sort than "B1") but "B2" without alcohol and "B3" stout beer. Furthermore, beer "B5" purchased in an aluminium can was also analysed to prove that a discrimination of the same beer sort from the same supplier brand filled in glass bottle "B4" and aluminium can is possible. The ethanol content ranged between 5.0 and $5.6 \%$ (v/v). The beer samples were cooled to $4{ }^{\circ} \mathrm{C}$ to minimize the loss of very volatile compounds. B1 beer (100\% malt; $5 \%, \mathrm{v} / \mathrm{v}$, alcohol) sorts in glass bottles $(330 \mathrm{ml})$ were used for the analytical method optimization.

Commercial Scotch whisky (SW) samples (40\%, v/v, alcohol), were purchased from a local store (Funchal, Madeira Island), and frozen at $-28^{\circ} \mathrm{C}$ until their analysis. Before extraction the volatile compounds, the samples were unfrozen at 3-4 ${ }^{\circ} \mathrm{C}$. SW5 commercial Scotch whisky (SW) samples (40\%, v/v, alcohol), was used for the analytical method optimization.

All alcoholic beverage samples were coded (Table 1) with a letter (WW representing white wine, B representing beer and SW representing Scotch whisky samples) and a number (1-5) representing different samples within the same alcoholic beverage.

The high ethanol concentration $(\approx 40 \%, v / v)$ of the whisky samples required dilution before the extraction procedure. Some authors $[30,31]$ have found that an increase in the ethanol content decreases the extraction efficiency. Ethanol is one of the major whisky constituents that can compete with the other volatiles in the extraction by the fibre. All whisky samples were adjusted to $13 \%$ (v/v) alcohol by dilution with deionised water before extraction. Beers were spiked with ethanol to adjust their ethanol content at $13 \%(\mathrm{v} / \mathrm{v})$. 


\subsection{Extraction of beverages VOCs and SVOCs by headspace SPME}

HS-SPME requires a previous optimization of the extraction parameters that can affect extraction efficiencies, in order to obtain high recoveries of VOCs and SVOCs. Some SPME parameters with influence in the extraction process were selected for optimization: fibre coating, extraction time, extraction temperature, ionic strength and sample stirring. The WW1 white wine, B1 beer and SW5 whisky samples, were selected as the matrix for comparison of the performance of the five fibres. The fibre that presented the most complete profile from alcoholic beverages VOCs and SVOCs, was chosen to optimize the sampling procedure and the operating conditions. Extraction studies were conducted to determine the profiles of adsorption/absorption kinetics. The samples were extracted for predetermined exposure times of 5, 30, 45, 60 and $80 \mathrm{~min}$. The extraction was carried out at $30^{\circ} \mathrm{C}$ (controlled temperature) for wine and beer and $40^{\circ} \mathrm{C}$ for whisky, and each measurement was repeated three times. Each alcoholic beverage was also tested at four heating temperatures (30, 40, 50 and $60^{\circ} \mathrm{C}$ ) with $60 \mathrm{~min}$ of fibre-exposed time. The volume ratio headspace/solution was 1:1. The PDMS, DVB/CAR/PDMS and CAR/PDMS fibres, the most suitable for isolation of VOCs and SVOCs from wine, beer and whisky samples, were subsequently chosen for further method development.

For headspace sampling, $30 \mathrm{~mL}$ of each beverage sample was transferred into a $60 \mathrm{~mL}$ glass vial, which corresponds to a ratio of the volume of the liquid phase to the headspace volume $(1 / \beta)$ of 1.0 and hermetically sealed. The extractions were carried out without adjust of $\mathrm{pH}$. The ionic strength was increased using $\mathrm{NaCl}(30 \%, w / v)$ to improve the extraction efficiency by decreasing the solubility of hydrophilic compounds in the aqueous phase. A Teflon septum and an aluminium cap sealed the vial that was subsequently placed in a thermostatic bath adjusted to desired temperature, under continuous stirring (containing a $25 \mathrm{~mm} \times 5 \mathrm{~mm}$ stirring bar) at $750 \mathrm{rpm}$ to promote the transference of the compounds from the sample to the headspace. After this step, the adequate SPME fibre was manually inserted into the sample vial headspace and then exposed to the headspace of the beverage samples and kept for $60 \mathrm{~min}$ at $30^{\circ} \mathrm{C}$ for white wine and beer samples isolation VOCs and SVOCs and $40^{\circ} \mathrm{C}$ for whisky samples. Blanks, corresponding to the analysis of the coating fibre not submitted to any extraction procedure, were run between a set of five analyses.

After sampling, the SPME fibre was withdrawn into the needle, removed from the vial and inserted into the hot injector port $\left(260^{\circ} \mathrm{C}\right)$ of the GC-qMSD system where the extracted analytes were thermally desorbed and transferred directly to the analytical column. Through all the work, a relatively long desorption time in the injector $(6 \mathrm{~min})$ was selected to avoid carryover between runs by ensuring full desorption of all analytes from the fibre. Each analysis was carried out four times.

\subsection{Gas chromatography-quadrupole mass spectrometry detection (GC-qMSD)}

The desorbed volatile compounds were separated in an Agilent $6890 \mathrm{~N}$ (Palo Alto, CA, USA) gas chromatograph system coupled to an Agilent 5975 quadrupole inert mass selective detector equipped with a $30 \mathrm{~m} \times 0.25 \mathrm{~mm}$ i.d., with a $0.25 \mu \mathrm{m}$ film thickness, BP-20 fused silica capillary column. Ultra-highpurity helium (Helium N60, Air Liquid, Portugal) was used as the carrier gas at a flow rate $\approx 1 \mathrm{~mL} \mathrm{~min}^{-1}$ (column-head pressure: 13 psi). Water and oxygen traps were installed on the carrier gas lines. An insert of $0.75 \mathrm{~mm}$ i.d. was used and the injector temperature was set according to the nature of the fibre used. This temperature close to the maximum temperature recommended by the manufacturer, allowed us to avoid a significant carry over effect. Splitless injections were used. The initial oven temperature program was $40^{\circ} \mathrm{C}$ holds for $1 \mathrm{~min}$ and then the temperature increased in three steps: $40-120^{\circ} \mathrm{C}$, at $2^{\circ} \mathrm{C} \mathrm{min}^{-1} ; 100-180^{\circ} \mathrm{C}$ at $1.7^{\circ} \mathrm{C} \mathrm{min}^{-1}$ and $180-220^{\circ} \mathrm{C}$, at $25^{\circ} \mathrm{C} \mathrm{min}^{-1}$. Each step was preceded by a small period at constant temperature for 2, 1 and $10 \mathrm{~min}$, respectively. The overall GC run time was $90.89 \mathrm{~min}$. Trap temperatures were as follows: manifold $180^{\circ} \mathrm{C}$, transfer line $220^{\circ} \mathrm{C}$, and trap $200^{\circ} \mathrm{C}$. The mass spectrometer was operated in electron ionization mode (EI) at $70 \mathrm{eV}$ and covering a mass-to-charge $(\mathrm{m} / \mathrm{z})$ range from 35 to 300 in full scan acquisition mode. The US National Institute of Standards and Technology (NIST05) mass spectral database library (Gaithersburg, MD, USA) was used for all tentative compound identifications. A reverse fit value of 850 was required for compounds to be considered a spectral match. Tentatively identified compounds also had to fit logically with respect to the retention time in the chromatograms. The Kováts retention indices were calculated for each peak and compared with the literature [32] in order to ensure the correct identification of the compounds. Based on the peak resolution, their areas were calculated from the total ion current. The resulting peak areas were expressed in arbitrary units of area.

\section{Results and discussion}

The first stage of this study addressed the optimization of the parameters that influence the aroma extraction by SPME. Among the several variables that could improve the SPME procedure, fibre coating, extraction temperature and extraction time, are three of the most important factors influencing the vapour pressure and equilibrium of the aroma compounds in the headspace, and therefore should be optimized. The results showed that, an increase of these factors results in an increase in the analytical signal. Sample agitation and ionic strength are other known factors that cause an increase in the method sensitivity.

Five different fibres were employed, namely PDMS, PA, CAR/PDMS, PDMS/DVB and DVB/CAR/PDMS. The beverages TIC chromatograms were compared in terms of total areas of the volatile compounds and number of compounds. The identified compounds were then organized in different groups accordingly to their chemical structure. This was done for $\mathrm{C}_{13}$-norisoprenoids/monoterpenes, sesquiterpenoids, higher alcohols, fatty acids, ethyl esters, furanic compounds, carbonyl compounds, aliphatic and aromatic hydrocarbons, while compounds of different structures were considered together as the class "miscellaneous". The method optimization enabled the evaluation of the effect of one variable at a time, keeping all the others constant during experiment. 
The comparison of the SPME fibre performance can be made in terms of extraction efficiency and number of identifiable compounds in the extracts. Therefore the normalized extraction efficiency, $N_{i, x}$ was defined as: $N=\sum A_{i, x} / \sum A_{i, F b e s t}$, where $\sum A_{i, x}$ is the sum of the $i$ peak area obtained with $x$ fibre, and $\sum A_{i, F b e s t}$ is the corresponding sum obtained after extraction the same matrix with the fibre that present the highest extraction efficiency.

From the relative composition of flavour volatiles of the alcoholic beverage, it can be concluded that ethyl esters, higher alcohols and fatty acids, major fermentation compounds, such as higher alcohols, ethyl esters and fatty acids constitute an important part of flavour of the studied alcoholic beverages, and probably play a significant role in their fruity notes. As predominant compounds 3-methyl-1-butanol, ethyl octanoate, ethyl decanoate and to a lesser extent, $\beta$-phenylethanol, isoamyl acetate, ethyl dodecanoate, ethyl acetate and octanoic acid, were found. The two fatty acid ethyl esters may contribute with sweet and fruity notes; 2and 3-methylbutan-1-ol should exhibit banana notes and $\beta$ phenylethanol may contribute with flowery, rose and honey notes. Some compounds were found out to be common in the studied beverages, such as 3-methyl-1-butanol, $\beta$ phenylethanol, isoamyl acetate, ethyl esters from C6, C8 and C10 fatty acids, and hexanoic, octanoic and decanoic acids.

\subsection{Method optimization}

\subsubsection{Selection of SPME fibre coating for different beverages}

The fibre coating is surely the most important parameter to be considered in a first stage, due to the influences on the chemical nature of the extracted analytes established by their characteristic polarity and volatility. To evaluate the extraction efficiency of volatile compounds from alcoholic beverages, and taking account of the physic/chemical characteristics of the targets under consideration, were tested five fibres (PDMS, PA, CAR/PDMS, PDMS/DVB and DVB/CAR/PDMS) among those used most routinely for assaying VOCs and SVOCs. Whereas PA and PDMS (liquid coatings) extract by absorption, the latter three can be considered as "solids" which extract by adsorption. At this evaluation stage, the extraction time was set at $60 \mathrm{~min}$ (in order to assure that the equilibrium could be established or a large amount of analytes would be extracted) and extraction temperature at $40^{\circ} \mathrm{C}$. The comparison of the SPME fibre performance was made in terms of extraction efficiency, number of identifiable compounds in the extract and reproducibility.

3.1.1.1. Wine. As shown in Fig. 1, the PDMS fibre allowed the best extraction efficiency for white wines VOCs and SVOCs. Under these conditions the DVB/CAR/PDMS fibre had a low sorption capacity. The results obtained using the five fibres on the same wine sample (WW1), in rigorously reproduced temperature and exposure time conditions, are reported in Table 2. The more polar fibre, PA, shows a more effective extraction for polar compounds such as higher alcohols and fatty acids while PDMS favours the extraction of less polar compounds like ethyl esters, monoterpenols/ $\mathrm{C}_{13}$-norisoprenoids, acetates and isoamyl esters. The profiles obtained for WW1 wine sample by
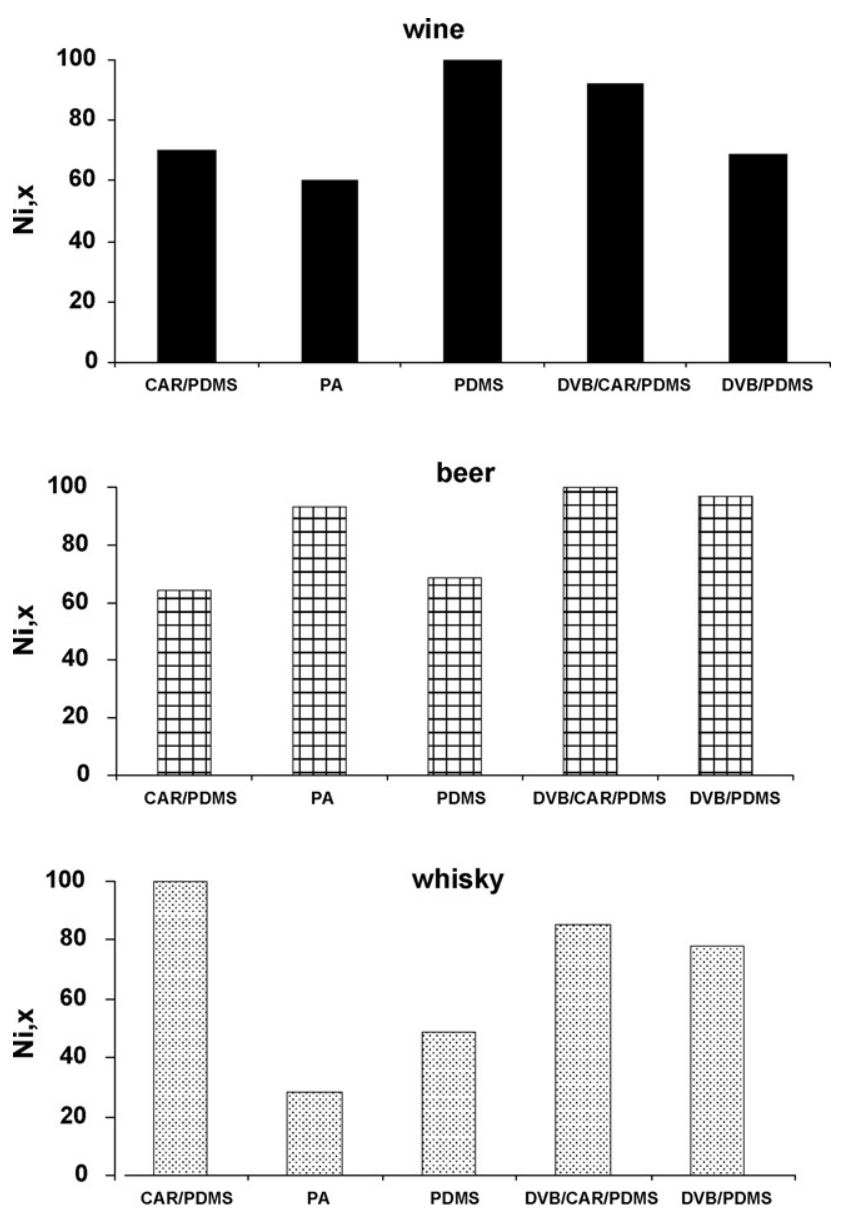

Fig. 1 - Normalized extraction efficiencies measured for CAR/PDMS, PA, PDMS, DVB/CAR/PDMS and DVB/PDMS fibres.

using different coatings in the same experimental conditions, shows that the fibres exhibit different extraction efficiency. The qualitative composition of white wine volatiles and the number of identified compounds using the five fibres under study is quite different. As seen in Table 2, a total of 40 and 39 compounds were identified using PA and CAR/PDMS coatings, whilst with PDMS/DVB, PDMS and DVB/CAR/PDMS coatings 32, 33 and 36 compounds were detected. The non-polar PDMS fibre allowed the best efficiency of extraction for volatile compounds, while under the same conditions, PA fibre showed the lowest sorption capacity. The former fibre provided the best sensitivity in terms of total compound peak areas and higher reproducibility; hence this fibre was chosen for evaluation of volatile compounds in white wines. DVB/CAR/PDMS fibre extracted $92.4 \%$ of PDMS while PA coating extracted the lowest amount (about $60.2 \%$ of PDMS). Similar amounts of volatiles (68.5-70.4\% of PDMS) were extracted when using either, PDMS/DVB or CAR/PDMS coatings (Fig. 1).

3.1.1.2. Beers. The most complete beer profile was obtained using the DVB/CAR/PDMS fibre, which enabled the detection of a wider range of compounds and produced higher signal intensities than the other coatings tested. The relative extraction efficiencies of VOCs and SVOCs from B1 beer with 
Table 2 - Volatile compounds identified in WW1 white wine after dynamic headspace solid-phase microextraction using different coatings (extraction temperature: $30^{\circ} \mathrm{C}$; extraction time: $60 \mathrm{~min} ; 30 \% \mathrm{NaCl} ; 750 \mathrm{rpm}$ )

\begin{tabular}{|c|c|c|c|c|c|c|c|c|}
\hline \multirow[t]{2}{*}{$\mathrm{RT}$ (min) } & \multirow[t]{2}{*}{$\mathrm{KI}^{\mathrm{a}}$} & \multirow[t]{2}{*}{ Compound } & \multirow[t]{2}{*}{ Identity $^{b}$} & \multicolumn{5}{|c|}{ SPME coating } \\
\hline & & & & PA & PDMS & Stableflex ${ }^{\mathrm{c}}$ & PDMS/DVB & CAR/PDMS \\
\hline 4.521 & 1078 & Ethyl butanoate & A, B & $\mathrm{x}$ & $\mathrm{x}$ & $\mathrm{x}$ & $\mathrm{x}$ & $\mathrm{x}$ \\
\hline 4.698 & 1086 & 1-Propanol & A, B & $\mathrm{x}$ & $\mathrm{x}$ & $\mathrm{x}$ & $\mathrm{x}$ & $\mathrm{x}$ \\
\hline 5.342 & 1114 & Ethyl isovalerate & A, B & & $\mathrm{x}$ & & & \\
\hline 6.368 & 1150 & 2-Methyl-1-propanol & A, B & $\mathrm{x}$ & $\mathrm{x}$ & $\mathrm{x}$ & $\mathrm{x}$ & $\mathrm{x}$ \\
\hline 6.907 & 1167 & Isoamyl acetate & A & $\mathrm{x}$ & $\mathrm{x}$ & $\mathrm{x}$ & $\mathrm{x}$ & $\mathrm{x}$ \\
\hline 8.185 & 1202 & 1-Butanol & A, B & $\mathrm{x}$ & & & & \\
\hline 10.804 & 1266 & 3-Methyl-1-butanol & B & $\mathrm{x}$ & $\mathrm{x}$ & $\mathrm{x}$ & $\mathrm{x}$ & $\mathrm{x}$ \\
\hline 12.104 & 1293 & Ethyl hexanoate & B & $\mathrm{x}$ & $\mathrm{x}$ & $\mathrm{x}$ & $\mathrm{x}$ & $\mathrm{x}$ \\
\hline 13.19 & 1316 & Styrene & A, B & & & $\mathrm{x}$ & & $\mathrm{x}$ \\
\hline 13.768 & 1328 & 1-Methyl-3-(1-methylethyl)-benzene & A, B & & & $\mathrm{x}$ & & $\mathrm{x}$ \\
\hline 14.389 & 1340 & Hexyl acetate & B & $x$ & $\mathrm{x}$ & $\mathrm{x}$ & $\mathrm{x}$ & $\mathrm{x}$ \\
\hline 16.638 & 1381 & 3-Methyl-cyclohexanone & A, B & & & $\mathrm{x}$ & & \\
\hline 18.129 & 1407 & (S)-(+)-3-Methyl-1-pentanol & B & $x$ & & & & \\
\hline 18.374 & 1411 & Ethyl heptanoate & B & $\mathrm{x}$ & $\mathrm{x}$ & $\mathrm{x}$ & $\mathrm{x}$ & $\mathrm{x}$ \\
\hline 19.22 & 1428 & Ethyl lactate & B & $\mathrm{x}$ & & & & \\
\hline 20.021 & 1443 & 1-Hexanol & A, B & $\mathrm{x}$ & $\mathrm{x}$ & $\mathrm{x}$ & $\mathrm{x}$ & $\mathrm{x}$ \\
\hline 20.704 & 1456 & (Z)-3-Hexen-1-ol & A, B & $\mathrm{x}$ & & & & \\
\hline 22.075 & 1479 & (E)-3-Hexen-1-ol & A, B & $\mathrm{x}$ & & $\mathrm{x}$ & & \\
\hline 22.384 & 1484 & Methyl octanoate & B & $\mathrm{x}$ & $x$ & $\mathrm{x}$ & $\mathrm{x}$ & $\mathrm{x}$ \\
\hline 24.427 & 1521 & Hexyl isobutanoate & B & & & & & $\mathrm{x}$ \\
\hline 25.872 & 1547 & Ethyl octanoate & A, B & $\mathrm{x}$ & $\mathrm{x}$ & $\mathrm{x}$ & $\mathrm{x}$ & $\mathrm{x}$ \\
\hline 27.644 & 1577 & Isopentyl hexanoate & A, B & $\mathrm{x}$ & $\mathrm{x}$ & $\mathrm{x}$ & $\mathrm{x}$ & $\mathrm{x}$ \\
\hline 27.863 & 1580 & Acetic acid & B & $\mathrm{x}$ & $\mathrm{x}$ & $\mathrm{x}$ & $\mathrm{x}$ & $\mathrm{x}$ \\
\hline 27.948 & 1582 & Furfural & A, B & & & & & $\mathrm{x}$ \\
\hline 28.347 & 1588 & (D)-Menthone & B & & & $\mathrm{x}$ & & \\
\hline 28.951 & 1597 & Octyl acetate & A, B & & $\mathrm{x}$ & & $\mathrm{x}$ & $\mathrm{x}$ \\
\hline 30.334 & 1619 & (S)-(+)-3-Hydroxytetrahydrofuran & B & $\mathrm{x}$ & & & & \\
\hline 31.739 & 1639 & 3-Ethyl-1,5-octadiene & B & & & & $\mathrm{x}$ & \\
\hline 32.308 & 1647 & Propyl octanoate & B & $\mathrm{x}$ & & $\mathrm{x}$ & & \\
\hline 33.625 & 1665 & Ethyl nonanoate & B & $\mathrm{x}$ & $x$ & $\mathrm{x}$ & $\mathrm{x}$ & $\mathrm{x}$ \\
\hline 34.887 & 1807 & Isobutyl octanoate & B & & $\mathrm{x}$ & $\mathrm{x}$ & $\mathrm{x}$ & $\mathrm{x}$ \\
\hline 35.018 & 1809 & Linalool & B & $\mathrm{x}$ & $\mathrm{x}$ & $\mathrm{x}$ & $\mathrm{x}$ & \\
\hline 35.573 & 1821 & 1-Octanol & B & $\mathrm{x}$ & $\mathrm{x}$ & $\mathrm{x}$ & & \\
\hline 38.048 & 1868 & Methyl nonanoate & A, B & $\mathrm{x}$ & & $\mathrm{x}$ & $\mathrm{x}$ & $\mathrm{x}$ \\
\hline 39.247 & 1890 & Hexyl hexanoate & A, B & & & & & $\mathrm{x}$ \\
\hline 39.898 & 1902 & Octyl butanoate & A, B & & & & & $\mathrm{x}$ \\
\hline 39.96 & 1904 & Ethyl 2-furoate & B & & & & & $\mathrm{x}$ \\
\hline 40.029 & 1905 & Pulegone & B & $\mathrm{x}$ & & & $\mathrm{x}$ & \\
\hline 41.49 & 1935 & Ethyl decanoate & A, B & $\mathrm{x}$ & $\mathrm{x}$ & $\mathrm{x}$ & $\mathrm{x}$ & $\mathrm{x}$ \\
\hline 42.679 & 1958 & (Z)-3-Hexenyl hexanoate & A, B & & & & & $\mathrm{x}$ \\
\hline 42.899 & 1962 & Isoamyl caprylate & A, B & $\mathrm{x}$ & $\mathrm{x}$ & $\mathrm{x}$ & $\mathrm{x}$ & $\mathrm{x}$ \\
\hline 43.346 & 1971 & 1-Nonanol & A, B & $\mathrm{x}$ & & & & \\
\hline 43.431 & 1973 & l-Isoasparagine & B & & & & & $\mathrm{x}$ \\
\hline 44.533 & 1993 & Diethyl succinate & A, B & $\mathrm{x}$ & $\mathrm{x}$ & $\mathrm{x}$ & $\mathrm{x}$ & $\mathrm{x}$ \\
\hline 45.356 & 2010 & Ethyl 9-decenoate & B & $\mathrm{x}$ & $\mathrm{x}$ & $\mathrm{x}$ & $\mathrm{x}$ & $\mathrm{x}$ \\
\hline 47.341 & 2049 & 1,1,6-Trimethyl-1,2-dihydronaphtalene & A, B & $\mathrm{x}$ & $\mathrm{x}$ & & & \\
\hline 51.027 & 2124 & 3-Methyl 1-hexanol & B & $\mathrm{x}$ & $\mathrm{x}$ & & & $\mathrm{x}$ \\
\hline 53.668 & 2181 & 2-Phenylethyl acetate & A, B & $\mathrm{x}$ & $\mathrm{x}$ & $\mathrm{x}$ & $\mathrm{x}$ & $\mathrm{x}$ \\
\hline 54.12 & 2191 & Hexyl octanoate & B & $\mathrm{x}$ & $\mathrm{x}$ & $\mathrm{x}$ & $\mathrm{x}$ & $\mathrm{x}$ \\
\hline 54.244 & 2194 & Octyl hexanoate & B & & & $\mathrm{x}$ & $\mathrm{x}$ & $\mathrm{x}$ \\
\hline 56.6 & 2238 & Ethyl dodecanoate & B & $\mathrm{x}$ & $\mathrm{x}$ & $\mathrm{x}$ & $\mathrm{x}$ & $\mathrm{x}$ \\
\hline 56.989 & 2555 & Hexanoic acid & B & $\mathrm{x}$ & $\mathrm{x}$ & $\mathrm{x}$ & $\mathrm{x}$ & $\mathrm{x}$ \\
\hline 60.454 & 2328 & Phenylethyl alcohol & A, B & $\mathrm{x}$ & $\mathrm{x}$ & $\mathrm{x}$ & $\mathrm{x}$ & $\mathrm{x}$ \\
\hline 61.177 & 2342 & 4,6-Di(1,1dimethylethyl)-2-methylphenol & A, B & $\mathrm{x}$ & $\mathrm{x}$ & & & \\
\hline 71.463 & 1524 & Octanoic acid & A, B & $\mathrm{x}$ & $\mathrm{x}$ & $\mathrm{x}$ & $\mathrm{x}$ & $\mathrm{x}$ \\
\hline 81.808 & 2761 & Decanoic acid & B & $\mathrm{x}$ & $\mathrm{x}$ & $\mathrm{x}$ & $\mathrm{x}$ & $\mathrm{x}$ \\
\hline Total com & unds i & ntified by fibre & & 40 & 33 & 36 & 32 & 39 \\
\hline
\end{tabular}

\footnotetext{
${ }^{a}$ Experimentally determined Kovàts indices on the BP-20 column, relative to $\mathrm{C}_{8}-\mathrm{C}_{20}$ hydrocarbons.

b A, components identified on the basis of the retention time and EI mass spectra of pure standard; B, components identified on the basis of their EI mass spectra only.

${ }^{c}$ DVB/CAR/PDMS coating.
} 
Table 3 - Volatile compounds identified in B1 beer after dynamic headspace solid-phase microextraction using different coatings (extraction temperature: $40^{\circ} \mathrm{C}$; extraction time: $60 \mathrm{~min} ; 30 \% \mathrm{NaCl} ; 750 \mathrm{rpm}$ )

$\begin{array}{llll}\mathrm{RT}(\mathrm{min}) & \mathrm{KI}^{\mathrm{a}} & \text { Compound } & \text { Identity } \\ \end{array}$

PA PDMS Stableflex ${ }^{c}$ PDMS/DVB CAR/PDMS

\begin{tabular}{|c|c|c|c|c|c|c|c|c|}
\hline 4.479 & 1076 & Ethyl butanoate & A, B & & $\mathrm{x}$ & $\mathrm{x}$ & & \\
\hline 4.59 & 1081 & Toluene & A, B & & & & & $\mathrm{x}$ \\
\hline 6.335 & 1149 & 2-Methyl-1-propanol & A, B & $\mathrm{x}$ & $\mathrm{x}$ & $\mathrm{x}$ & $\mathrm{x}$ & $\mathrm{x}$ \\
\hline 6.872 & 1166 & Isoamyl acetate & A, B & $\mathrm{x}$ & $\mathrm{x}$ & $\mathrm{x}$ & $\mathrm{x}$ & $\mathrm{x}$ \\
\hline 9.575 & 1238 & Trans-1,2-bis(1-methylethenyl)-ciclobutane & A & & & $\mathrm{x}$ & & \\
\hline 10.889 & 1268 & 3-Methyl-1-butanol & A, B & $\mathrm{x}$ & $\mathrm{x}$ & $\mathrm{x}$ & $\mathrm{x}$ & $\mathrm{x}$ \\
\hline 12.098 & 1293 & Ethyl hexanoate & B & $\mathrm{x}$ & $\mathrm{x}$ & $\mathrm{x}$ & $\mathrm{x}$ & $\mathrm{x}$ \\
\hline 13.442 & 1321 & 3-Ethylpentane & B & & & & & $\mathrm{x}$ \\
\hline 13.546 & 1323 & 2-Ethyl-1,4-dimethyl-benzene & B & $x$ & $\mathrm{x}$ & & $\mathrm{x}$ & $\mathrm{x}$ \\
\hline 14.389 & 1340 & Hexyl acetate & A, B & & $\mathrm{x}$ & $\mathrm{x}$ & $\mathrm{x}$ & $\mathrm{x}$ \\
\hline 14.968 & 1351 & (E)-2-Hexene & A, B & & & & $\mathrm{x}$ & \\
\hline 16.638 & 1381 & 3-Methylcyclohexanone & B & & & & $\mathrm{x}$ & \\
\hline 18.139 & 1407 & Ethyl valerate & A, B & & & & $\mathrm{x}$ & $\mathrm{x}$ \\
\hline 18.374 & 1411 & Ethyl heptanoate & B & & $\mathrm{x}$ & $\mathrm{x}$ & $\mathrm{x}$ & \\
\hline 21.283 & 1466 & Heptyl acetate & B & $\mathrm{x}$ & $\mathrm{x}$ & $\mathrm{x}$ & $\mathrm{x}$ & $\mathrm{x}$ \\
\hline 22.172 & 1481 & (E)-2-Nonen-1-ol & B & & & & $\mathrm{x}$ & $\mathrm{x}$ \\
\hline 22.394 & 1485 & 1-Nonanol & A, B & & & $\mathrm{x}$ & & \\
\hline 22.424 & 1485 & 3-Heptene & A, B & & & $\mathrm{x}$ & & \\
\hline 23.963 & 1512 & Hexyl isobutyrate & B & & & & & $x$ \\
\hline 25.669 & 1543 & Ethyl octanoate & A, B & $x$ & $\mathrm{x}$ & $x$ & $\mathrm{x}$ & $\mathrm{x}$ \\
\hline 26.225 & 1553 & (2S)-Trans-MMCH ${ }^{\mathrm{d}}$ & B & & & & $\mathrm{x}$ & \\
\hline 27.81 & 1579 & Guaiacol & A, B & $\mathrm{x}$ & $\mathrm{x}$ & $\mathrm{x}$ & $\mathrm{x}$ & $\mathrm{x}$ \\
\hline 28.248 & 1586 & Trans-MMCH ${ }^{\mathrm{e}}$ & A, B & & & & $\mathrm{x}$ & \\
\hline 28.951 & 1597 & Octyl acetate & B & & $\mathrm{x}$ & $\mathrm{x}$ & $\mathrm{x}$ & $\mathrm{x}$ \\
\hline 30.154 & 1616 & Decanal & A, B & & & & $\mathrm{x}$ & $\mathrm{x}$ \\
\hline 30.164 & 1616 & (E)-2-Hexen-1-ol & B & $\mathrm{x}$ & & & & \\
\hline 30.376 & 1619 & 2-Ethyl-1-hexanol & A, B & $\mathrm{x}$ & $\mathrm{x}$ & $\mathrm{x}$ & $\mathrm{x}$ & $\mathrm{x}$ \\
\hline 33.469 & 1663 & 4-Cyclohexylresorcinol & B & & & $\mathrm{x}$ & & \\
\hline 33.508 & 1664 & Ethyl valerate & B & & $\mathrm{x}$ & & & \\
\hline 34.969 & 1809 & 3,5-Dimethylcyclohexanol & B & & & & $\mathrm{x}$ & \\
\hline 35.537 & 1820 & 1-Hexene & B & & $\mathrm{x}$ & & $\mathrm{x}$ & \\
\hline 35.587 & 1821 & 1-Octanol & B & & & & $\mathrm{x}$ & \\
\hline 33.596 & 1665 & Ethyl nonanoate & B & & & & & \\
\hline 35.001 & 1810 & Linalool & B & & & $\mathrm{x}$ & & \\
\hline 35.525 & 1820 & Cis-1,2-diethylcyclobutane & B & $\mathrm{x}$ & & $\mathrm{x}$ & & $\mathrm{x}$ \\
\hline 35.586 & 1821 & 1,2-Diethyl-cyclobutane & A, B & & $\mathrm{x}$ & $\mathrm{x}$ & & $\mathrm{x}$ \\
\hline 39.084 & 1887 & Hexyl hexanoate & A, B & $\mathrm{x}$ & & & & $\mathrm{x}$ \\
\hline 39.931 & 1903 & $\mathrm{MMCH}^{\mathrm{f}}$ & A, B & & & & $\mathrm{x}$ & \\
\hline 41.269 & 1930 & Ethyl decanoate & B & $\mathrm{x}$ & $\mathrm{x}$ & $\mathrm{x}$ & $\mathrm{x}$ & $\mathrm{x}$ \\
\hline 42.424 & 1953 & Ethyl benzoate & B & & & $\mathrm{x}$ & $\mathrm{x}$ & $\mathrm{x}$ \\
\hline 42.925 & 1963 & Isoamyl caprylate & A, B & & $\mathrm{x}$ & & $\mathrm{x}$ & \\
\hline 43.14 & 1967 & Citronellyl butyrate & A, B & & $\mathrm{x}$ & $\mathrm{x}$ & $\mathrm{x}$ & $\mathrm{x}$ \\
\hline 43.301 & 1970 & 3-Methyl-1-pentene & A, B & & & & & $\mathrm{x}$ \\
\hline 43.448 & 1973 & 4-Methyl-1-heptanol & A, B & & $\mathrm{x}$ & & $\mathrm{x}$ & \\
\hline 43.484 & 1974 & 2-Furanmethanol & B & & & $\mathrm{x}$ & & \\
\hline 43.546 & 1975 & 2-Methyl-2,3-hexadiene & A, B & & & & & $\mathrm{x}$ \\
\hline 44.167 & 1987 & 3-Methylpentanoic acid & $\mathrm{B}$ & $\mathrm{x}$ & $\mathrm{x}$ & $\mathrm{x}$ & $\mathrm{x}$ & \\
\hline 44.49 & 1993 & 2-methyl-1-pentanol & A, B & $\mathrm{x}$ & & & & $\mathrm{x}$ \\
\hline 44.647 & 1995 & Decyl acetate & B & & $\mathrm{x}$ & $\mathrm{x}$ & $\mathrm{x}$ & \\
\hline 45.415 & 2011 & Ethyl 9-decenoate & A, B & $\mathrm{x}$ & $\mathrm{x}$ & $\mathrm{x}$ & $\mathrm{x}$ & $\mathrm{x}$ \\
\hline 50.995 & 2123 & 1-Decanol & $\mathrm{B}$ & $\mathrm{x}$ & $\mathrm{x}$ & $\mathrm{x}$ & $\mathrm{x}$ & $\mathrm{x}$ \\
\hline 51.021 & 2123 & Cis-1-butyl-methylcyclopropane & B & & $\mathrm{x}$ & & $\mathrm{x}$ & \\
\hline 53.679 & 2182 & 2-Phenyl ethyl acetate & B & $x$ & $\mathrm{x}$ & $\mathrm{x}$ & $\mathrm{x}$ & $\mathrm{x}$ \\
\hline 56.342 & 2240 & Ethyl undecanoate & B & $\mathrm{x}$ & & $\mathrm{x}$ & & \\
\hline 56.565 & 2245 & Ethyl dodecanoate & A, B & $\mathrm{x}$ & $\mathrm{x}$ & $\mathrm{x}$ & $\mathrm{x}$ & $\mathrm{x}$ \\
\hline 57.025 & 2255 & Hexanoic acid & A, B & $\mathrm{x}$ & $\mathrm{x}$ & $\mathrm{x}$ & $\mathrm{x}$ & $\mathrm{x}$ \\
\hline 57.787 & 2272 & Amyl butyrate & A, B & & & & $\mathrm{x}$ & \\
\hline 58.372 & 2284 & TCIPPAg & B & & & & $\mathrm{x}$ & \\
\hline 60.438 & 2327 & Phenylethyl alcohol & A, B & $\mathrm{x}$ & $\mathrm{x}$ & $\mathrm{x}$ & $\mathrm{x}$ & $\mathrm{x}$ \\
\hline 62.569 & 2370 & DMEOPh & B & & $\mathrm{x}$ & & & \\
\hline 63.821 & 2395 & Phenylethyl isobutyrate & B & $\mathrm{x}$ & $\mathrm{x}$ & $\mathrm{x}$ & $\mathrm{x}$ & $\mathrm{x}$ \\
\hline
\end{tabular}


Table 3 (Continued)

\begin{tabular}{|c|c|c|c|c|c|c|c|c|}
\hline \multirow[t]{2}{*}{$\mathrm{RT}(\mathrm{min})$} & \multirow[t]{2}{*}{$\mathrm{KI}^{\mathrm{a}}$} & \multirow[t]{2}{*}{ Compound } & \multirow[t]{2}{*}{ Identity } & \multicolumn{5}{|c|}{ SPME coating } \\
\hline & & & & PA & PDMS & Stableflex ${ }^{c}$ & PDMS/DVB & CAR/PDMS \\
\hline 65.38 & 2426 & 3,5-Dimethyl-1-hexene & A, B & $\mathrm{x}$ & & & & \\
\hline 65.635 & 2432 & Camphene & A, B & & & & $\mathrm{x}$ & \\
\hline 70.247 & 2512 & $\beta$-Myrcene & A, B & & & & $\mathrm{x}$ & \\
\hline 70.293 & 2512 & Linalyl acetate & A & $\mathrm{x}$ & & & & \\
\hline 71.569 & 2525 & Octanoic acid & A, B & $\mathrm{x}$ & $\mathrm{x}$ & $\mathrm{x}$ & $\mathrm{x}$ & $\mathrm{x}$ \\
\hline 74.418 & 2608 & Ethyl cinnamate & A & $\mathrm{x}$ & & & $\mathrm{x}$ & \\
\hline 78.259 & 2686 & 2-Methoxy-4-vinylphenol & A, B & $\mathrm{x}$ & $\mathrm{x}$ & $\mathrm{x}$ & $\mathrm{x}$ & $\mathrm{x}$ \\
\hline 78.301 & 2687 & 2'-Hydroxy-5'-methylacetophenone & A & & & & & $\mathrm{x}$ \\
\hline 81.772 & 2760 & Decanoic acid & A, B & $\mathrm{x}$ & $\mathrm{x}$ & $\mathrm{x}$ & $\mathrm{x}$ & $\mathrm{x}$ \\
\hline 84.083 & 2803 & 9-Decenoic acid & A & $\mathrm{x}$ & & & & \\
\hline 84.188 & 2805 & $\mathrm{CPT}^{\mathrm{i}}$ & A & & $\mathrm{x}$ & & $\mathrm{x}$ & $\mathrm{x}$ \\
\hline 85.035 & 2823 & (E)-3-Penten-2-one & A & $\mathrm{x}$ & & & & \\
\hline 88.421 & 2891 & Dodecanoic acid & A, B & $\mathrm{x}$ & $\mathrm{x}$ & $\mathrm{x}$ & $\mathrm{x}$ & \\
\hline 88.545 & 2893 & 5-Hydroxymethyl)furfural & A, B & & $\mathrm{x}$ & $\mathrm{x}$ & & \\
\hline 89.238 & 2907 & 4-Chloro-2-nitrophenol & B & & $\mathrm{x}$ & & & $\mathrm{x}$ \\
\hline \multicolumn{4}{|c|}{ Total compounds identified per fiber } & 32 & 38 & 38 & 48 & 39 \\
\hline \multicolumn{9}{|c|}{$\begin{array}{l}\text { a Experimentally determined Kovàts indices on the BP- } 20 \text { column, relative to } \mathrm{C}_{8}-\mathrm{C}_{20} \text { hydrocarbons. } \\
\text { b A, components identified on the basis of the retention time and EI mass spectra of pure standard; B, components identified on the basis of } \\
\text { their EI mass spectra only. }\end{array}$} \\
\hline \multicolumn{9}{|c|}{ c DVB/CAR/PDMS coating. } \\
\hline \multicolumn{9}{|c|}{ d (2S-Trans)-5-methyl-2-(1-methylethyl)cyclohexanone. } \\
\hline \multicolumn{9}{|c|}{ e Trans-5-methyl-2-(1-methylethyl)cyclohexanone. } \\
\hline \multicolumn{9}{|c|}{ f 5-Methyl-2-(1-methylethyl)cyclohexanone. } \\
\hline \multicolumn{9}{|c|}{ g 2,2,4-Trimethyl-3-carboxyisopropyl pentanoic acid isobutyl ester. } \\
\hline \multicolumn{9}{|c|}{ h 2,6-Bis(1,1-dimethylethyl)-4-(1-oxopropyl)phenol. } \\
\hline i $(1 \alpha, 2 \beta, 3 \beta$ & $-1,2,3$ & Cyclopentanetetrol. & & & & & & \\
\hline
\end{tabular}

different coatings are summarized in Fig. 1. The extraction efficiency of DVB/CAR/PDMS fibre was set as $100 \%$. The PA and PDMS/DVB (good selectivity for middle- to high-molecular weight analytes) exhibit similar sensitivities. Under these conditions, the PDMS fibre shows a low sorption capacity for B1 beer VOCs and SVOCs. As seen in Table 3, a total of 77 compounds were identified in B1 beer using the five fibres under study. Thirty-three compounds were identified using PA fibre whilst PDMS and DVB/CAR/PDMS extracted the same number of compounds, 38. With the PDMS/DVB and CAR/PDMS coatings it was possible to identify 48 and 39 compounds, respectively.

3.1.1.3. Whisky. The most complete profile of whisky VOCs and SVOCs corresponds to analyses carried with the CAR/PDMS fibre, which extracted more than 55 compounds (esters, aldehydes, alcohols, ketones, acids, ethers, aromatic compounds, alicyclic compounds, heterocyclic compounds, and hydrocarbons), at $40^{\circ} \mathrm{C}$ with a wide range of polarities and molecular masses (Table 4).

Higher alcohols have a larger affinity for PA fibre. This coating also presents better sensitivity for furanic compounds, carbonyl compounds and miscellaneous. DVB/CAR/PDMS coating showed a strong extraction capacity for aliphatic and aromatic hydrocarbons, $\mathrm{C}_{13}$-norisoprenoids/monoterpenes and ethyl esters. The most intense peak in the SW5 sample corresponds to ethyl octanoate. This compound was detected in all used fibres. As shown in Table 5, higher alcohols, low-chain esters and C8, C10 and C12, fatty acids were also detected. The PDMS fibre extracted $48.58 \%$ of CAR/PDMS while PA coating extracted the lowest amount (about $28.7 \%$ of CAR/PDMS). Similar amounts of volatiles $(78.5-85.6 \%$ of CAR/PDMS) were extracted when using either, PDMS/DVB or DVB/CAR/PDMS coatings (Fig. 1).

\subsubsection{Effect of extraction time}

The extraction time depends on the chemical nature of the target compounds, but in particular on the polymeric phase. In order to investigate the sorption behaviour, a time profile of adsorption of the different class of compounds onto the $100 \mu \mathrm{m}$ PDMS for wines, the $50 / 30 \mu \mathrm{m}$ DVB/CAR/PDMS for beers and the $75 \mu \mathrm{m}$ CAR/PDMS for whiskys was determined in order to assess the optimum SPME sampling period. Different extraction times ranging from 5 to 80 min namely, 5, $30,45,60$ and $80 \mathrm{~min}$, were examined for $30 \mathrm{ml}$ of beverage sample at $30^{\circ} \mathrm{C}$. In Fig. 2, the MS response against the SPME sampling period is displayed for CAR/PDMS fibre after different extraction times (5-80 min). This figure shows the efficiency of the extraction displayed as the sum of volatile compounds total peak areas after different exposure times of the fibre to the beverage headspace for 5, 30, 45, 60 and $80 \mathrm{~min}$. An exposure time of $60 \mathrm{~min}$ was chosen for VOCs and SVOCs extraction from wine, beer and whisky samples.

It was observed that a typical extraction profile consists of an initial rapid portioning followed by a slower prolonged uptake and finally a steady-state equilibrium between the fibre and the vapour phase of the analyte. As it can be verified there is an increase of the total free fraction throughout the extrac- 
Table 4 - Volatile compounds identified in SW5 whisky after dynamic headspace solid-phase microextraction using different coatings (extraction temperature: $40^{\circ} \mathrm{C}$; extraction time: $60 \mathrm{~min} ; 30 \% \mathrm{NaCl} ; 750 \mathrm{rpm}$ )

\begin{tabular}{|c|c|c|c|c|c|c|c|c|}
\hline \multirow[t]{2}{*}{$\mathrm{RT}$ (min) } & \multirow[t]{2}{*}{$\mathrm{KI}^{\mathrm{a}}$} & \multirow[t]{2}{*}{ Compound } & \multirow[t]{2}{*}{ Identity $^{\mathrm{b}}$} & \multicolumn{5}{|c|}{ SPME coating } \\
\hline & & & & PA & PDMS & Stableflex ${ }^{\mathrm{c}}$ & PDMS/DVB & CAR/PDMS \\
\hline 4.521 & 1078 & Ethyl butanoate & A, B & $\mathrm{x}$ & $\mathrm{x}$ & $\mathrm{x}$ & $\mathrm{x}$ & $\mathrm{x}$ \\
\hline 4.698 & 1086 & 1-Propanol & A, B & $\mathrm{x}$ & $\mathrm{x}$ & $\mathrm{x}$ & $\mathrm{x}$ & $\mathrm{x}$ \\
\hline 5.342 & 1114 & Ethyl isovalerate & A, B & & $\mathrm{x}$ & & & \\
\hline 6.368 & 1150 & 2-Methyl-1-propanol & A, B & $\mathrm{x}$ & $\mathrm{x}$ & $\mathrm{x}$ & $\mathrm{x}$ & $\mathrm{x}$ \\
\hline 6.907 & 1167 & Isoamyl acetate & A & $\mathrm{x}$ & $\mathrm{x}$ & $\mathrm{x}$ & $\mathrm{x}$ & $\mathrm{x}$ \\
\hline 8.185 & 1202 & 1-Butanol & A, B & $\mathrm{x}$ & & & & \\
\hline 10.804 & 1266 & 3-Methyl-1-butanol & B & $\mathrm{x}$ & $\mathrm{x}$ & $\mathrm{x}$ & $\mathrm{x}$ & $\mathrm{x}$ \\
\hline 12.104 & 1293 & Ethyl hexanoate & B & $\mathrm{x}$ & $\mathrm{x}$ & $\mathrm{x}$ & $\mathrm{x}$ & $\mathrm{x}$ \\
\hline 13.19 & 1316 & Styrene & A, B & & & $\mathrm{x}$ & & $\mathrm{x}$ \\
\hline 13.768 & 1328 & 1-Methyl-3-(1-methylethyl)-benzene & A, B & & & $\mathrm{x}$ & & $\mathrm{x}$ \\
\hline 14.389 & 1340 & Hexyl acetate & B & $\mathrm{x}$ & $\mathrm{x}$ & $\mathrm{x}$ & $\mathrm{x}$ & $\mathrm{x}$ \\
\hline 16.638 & 1381 & 3-Methyl-cyclohexanone & A, B & & & $\mathrm{x}$ & & \\
\hline 18.129 & 1407 & (S)-(+)-3-Methyl-1-pentanol & B & $\mathrm{x}$ & & & & \\
\hline 18.374 & 1411 & Ethyl heptanoate & B & $\mathrm{x}$ & $\mathrm{x}$ & $\mathrm{x}$ & $\mathrm{x}$ & $\mathrm{x}$ \\
\hline 19.22 & 1428 & Ethyl lactate & B & $\mathrm{x}$ & & & & \\
\hline 20.021 & 1443 & 1-Hexanol & A, B & $\mathrm{x}$ & $\mathrm{x}$ & $\mathrm{x}$ & $\mathrm{x}$ & $\mathrm{x}$ \\
\hline 20.704 & 1456 & (Z)-3-Hexen-1-ol & A, B & $\mathrm{x}$ & & & & \\
\hline 22.075 & 1479 & (E)-3-Hexen-1-ol & A, B & $\mathrm{x}$ & & $\mathrm{x}$ & & \\
\hline 22.384 & 1484 & Methyl octanoate & B & $\mathrm{x}$ & $\mathrm{x}$ & $\mathrm{x}$ & $\mathrm{x}$ & $\mathrm{x}$ \\
\hline 24.427 & 1521 & 2-Methylhexyl propanoate & B & & & & & $\mathrm{x}$ \\
\hline 25.872 & 1547 & Ethyl octanoate & A, B & $\mathrm{x}$ & $\mathrm{x}$ & $\mathrm{x}$ & $\mathrm{x}$ & $\mathrm{x}$ \\
\hline 27.644 & 1577 & Isopentyl hexanoate & A, B & $\mathrm{x}$ & $\mathrm{x}$ & $\mathrm{x}$ & $\mathrm{x}$ & $\mathrm{x}$ \\
\hline 27.863 & 1580 & Acetic acid & B & $\mathrm{x}$ & $\mathrm{x}$ & $\mathrm{x}$ & $\mathrm{x}$ & $\mathrm{x}$ \\
\hline 27.948 & 1582 & Furfural & A, B & & & & & $\mathrm{x}$ \\
\hline 28.347 & 1588 & (D)-Menthone & B & & & $\mathrm{x}$ & & \\
\hline 28.951 & 1597 & Octyl acetate & A, B & & $\mathrm{x}$ & & $\mathrm{x}$ & $\mathrm{x}$ \\
\hline 30.334 & 1619 & (S)-(+)-3-Hydroxytetrahydrofuran & $\mathrm{B}$ & $\mathrm{x}$ & & & & \\
\hline 31.739 & 1639 & 3-Ethyl-1,5-octadiene & B & & & & $\mathrm{x}$ & \\
\hline 32.308 & 1647 & Propyl octanoate & B & $\mathrm{x}$ & & $\mathrm{x}$ & & \\
\hline 33.619 & 1665 & Ethyl octanoate & B & & $\mathrm{x}$ & & $\mathrm{x}$ & $\mathrm{x}$ \\
\hline 33.625 & 1665 & Ethyl nonanoate & B & $\mathrm{x}$ & $\mathrm{x}$ & $\mathrm{x}$ & $\mathrm{x}$ & $\mathrm{x}$ \\
\hline 34.887 & 1807 & Isobutyl octanoate & B & & $\mathrm{x}$ & $\mathrm{x}$ & $\mathrm{x}$ & $\mathrm{x}$ \\
\hline 35.018 & 1809 & Linalool & B & $\mathrm{x}$ & $\mathrm{x}$ & $\mathrm{x}$ & $\mathrm{x}$ & \\
\hline 35.573 & 1821 & 1-Octanol & B & $\mathrm{x}$ & $\mathrm{x}$ & $\mathrm{x}$ & & \\
\hline 38.048 & 1868 & Methyl nonanoate & A, B & $\mathrm{x}$ & & $\mathrm{x}$ & $\mathrm{x}$ & $\mathrm{x}$ \\
\hline 39.247 & 1890 & Hexyl hexanoate & A, B & & & & & $\mathrm{x}$ \\
\hline 39.898 & 1902 & Octyl butanoate & A, B & & & & & $\mathrm{x}$ \\
\hline 39.96 & 1904 & Ethyl 2-furoate & B & & & & & $\mathrm{x}$ \\
\hline 40.029 & 1905 & Pulegone & B & $\mathrm{x}$ & & & $\mathrm{x}$ & \\
\hline 41.49 & 1935 & Ethyl decanoate & A, B & $\mathrm{x}$ & $\mathrm{x}$ & $\mathrm{x}$ & $\mathrm{x}$ & $\mathrm{x}$ \\
\hline 42.679 & 1958 & Ethyl benzoate & A, B & & & & & $\mathrm{x}$ \\
\hline 42.899 & 1962 & Isoamyl caprylate & A, B & $\mathrm{x}$ & $\mathrm{x}$ & $\mathrm{x}$ & $\mathrm{x}$ & $\mathrm{x}$ \\
\hline 43.346 & 1971 & 1-Nonanol & A, B & $\mathrm{x}$ & & & & \\
\hline 43.431 & 1973 & l-Isoasparagine & B & & & & & $\mathrm{x}$ \\
\hline 44.533 & 1993 & Diethyl succinate & A, B & $\mathrm{x}$ & $\mathrm{x}$ & $\mathrm{x}$ & $\mathrm{x}$ & $\mathrm{x}$ \\
\hline 45.356 & 2010 & Ethyl 9-decenoate & $\mathrm{B}$ & $\mathrm{x}$ & $\mathrm{x}$ & $\mathrm{x}$ & $\mathrm{x}$ & $\mathrm{x}$ \\
\hline 47.341 & 2049 & 1,1,6-Trimethyl-1,2-dihydronaphtalene & A, B & $\mathrm{x}$ & $\mathrm{x}$ & & & \\
\hline 51.027 & 2124 & 3-Methyl 1-hexanol & B & $\mathrm{x}$ & $\mathrm{x}$ & & & $\mathrm{x}$ \\
\hline 53.668 & 2181 & 2-Phenylethyl acetate & A, B & $\mathrm{x}$ & $\mathrm{x}$ & $\mathrm{x}$ & $\mathrm{x}$ & $\mathrm{x}$ \\
\hline 54.12 & 2191 & Hexyl octanoate & B & $\mathrm{x}$ & $\mathrm{x}$ & $\mathrm{x}$ & $\mathrm{x}$ & $\mathrm{x}$ \\
\hline 54.244 & 2194 & Octyl hexanoate & B & & & $\mathrm{x}$ & $\mathrm{x}$ & $\mathrm{x}$ \\
\hline 56.6 & 2238 & Ethyl dodecanoate & B & $\mathrm{x}$ & $\mathrm{x}$ & $\mathrm{x}$ & $\mathrm{x}$ & $\mathrm{x}$ \\
\hline 56.989 & 2555 & Hexanoic acid & B & $\mathrm{x}$ & $\mathrm{x}$ & $\mathrm{x}$ & $\mathrm{x}$ & $\mathrm{x}$ \\
\hline 60.454 & 2328 & Phenylethyl alcohol & A, B & $\mathrm{x}$ & $\mathrm{x}$ & $\mathrm{x}$ & $\mathrm{x}$ & $\mathrm{x}$ \\
\hline 61.177 & 2342 & 4,6-Di(1,1dimethylethyl)-2-methylphenol & A, B & $\mathrm{x}$ & $\mathrm{x}$ & & & \\
\hline 71.463 & 1524 & Octanoic acid & A, B & $\mathrm{x}$ & $\mathrm{x}$ & $\mathrm{x}$ & $\mathrm{x}$ & $\mathrm{x}$ \\
\hline 81.808 & 2761 & Decanoic acid & B & $\mathrm{x}$ & $\mathrm{x}$ & $\mathrm{x}$ & $\mathrm{x}$ & $\mathrm{x}$ \\
\hline \multicolumn{4}{|c|}{ Total compounds identified per fiber } & 40 & 34 & 36 & 33 & 40 \\
\hline
\end{tabular}

${ }^{a}$ Experimentally determined Kovàts indices on the BP-20 column, relative to $C_{8}-C_{20}$ hydrocarbons.

${ }^{b} \mathrm{~A}$, components identified on the basis of the retention time and EI mass spectra of pure standard; B, components identified on the basis of their EI mass spectra only.

c DVB/CAR/PDMS coating. 
Table 5 - Optimized conditions to extract VOCs and SVOCs from alcoholic beverages using HS-SPME procedure

\begin{tabular}{|c|c|c|c|}
\hline SPME & Wine & Beer & Whisky \\
\hline Fibre coating & PDMS & DVB/CAR/PDMS & CAR/PDMS \\
\hline Sampling temperature & $30^{\circ} \mathrm{C}$ & $30^{\circ} \mathrm{C}$ & $40^{\circ} \mathrm{C}$ \\
\hline Extraction time & $60 \mathrm{~min}$, under constant stirring velocity (750 rpm) & & \\
\hline Salt addition & $\mathrm{NaCl}, 30 \%(\mathrm{w} / \mathrm{v})$ & & \\
\hline Sample volume & $30 \mathrm{~mL}$ & & \\
\hline Vial volume & $60 \mathrm{~mL}$ & & \\
\hline \multicolumn{4}{|l|}{ GC-qMS } \\
\hline GC column & $30 \mathrm{~m} \times 0.5 \mathrm{~mm}$ i.d. $\times 0.25 \mu \mathrm{m}$ & & \\
\hline Injection temperature & $250^{\circ} \mathrm{C}$ & $260^{\circ} \mathrm{C}$ & $260^{\circ} \mathrm{C}$ \\
\hline Injection mode & Splitless & & \\
\hline GC oven gradient & Section 2.4 & & \\
\hline Carrier gas & Helium at constant flow $\left(1 \mathrm{~mL} \mathrm{~min}^{-1}\right)$ & & \\
\hline Transfer line temperature & $220^{\circ} \mathrm{C}$ & & \\
\hline Mass analyser & Quadrupole & & \\
\hline Ionization type & Electron impact (EI) & & \\
\hline Ionization energy & $70 \mathrm{eV}$ & & \\
\hline Ion source temperature & $200^{\circ} \mathrm{C}$ & & \\
\hline Detector voltage & Set at the autotune value $(1700-1800 \mathrm{~V})$ & & \\
\hline Mass fragment collected & $m / z=35-300$ & & \\
\hline Data acquisition rate & 50 spectra s $^{-1}$ & & \\
\hline
\end{tabular}

tion time. Ethyl esters and higher alcohols, reach balance in $30 \mathrm{~min}$, whereas the acetates, norisoprenoids, acetates, isoamyl esters only at $60 \mathrm{~min}$. For most volatiles, equilibrium of adsorption was reached between 45 and $60 \mathrm{~min}$, while for some other components the equilibrium was still not reached after $60 \mathrm{~min}$. It was also observed that the extracted amount decrease after 60 min sampling, most probably due to competition effects. Therefore, it can be concluded that the highest recovery was obtained after $60 \mathrm{~min}$, although the reproducibility was higher after an extraction time of $30 \mathrm{~min}$. Sixty minutes were selected as an adequate extraction time because some analytes had already reached equilibrium and the obtained sensitivity for the other analytes was acceptable.

\subsubsection{Effect of extraction temperature}

The SPME process is greatly influenced by temperature. Heating the sample to an elevated temperature, increases the release of analytes from the matrix and, therefore, increasing their concentration in the headspace. The analyte diffusion

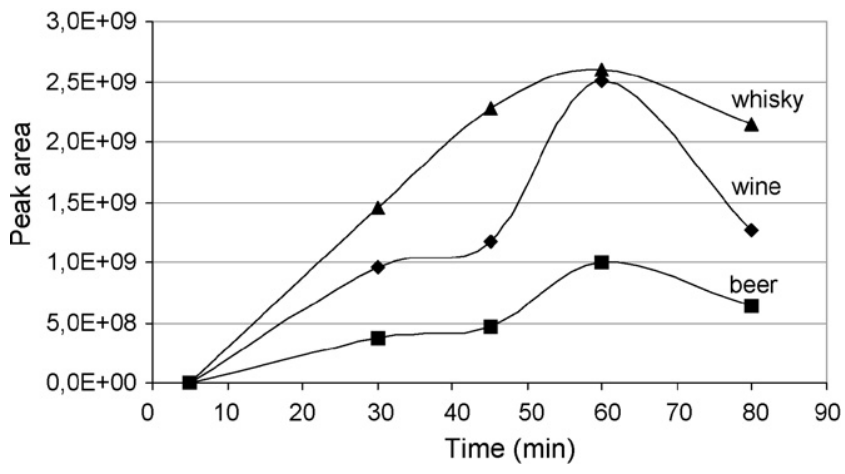

Fig. 2 - Effect of adsorption time at $30^{\circ} \mathrm{C}$ on the extraction efficiency of VOCs and SVOCs from alcoholic beverages by HS-SPME extraction using the best fibre for each beverage ( $y$-axis: total volatile compounds expressed as peak area).

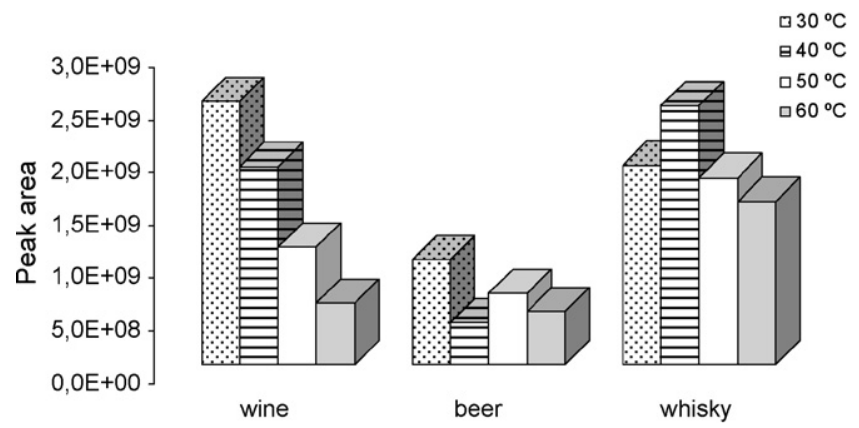

Fig. 3 - Influence of the extraction temperature on absorption of different wine, beer and whisky VOCs and SVOCs during HS-SPME extraction with the fibre that present the most complete profile in each sample, PDMS for wines, DVB/CAR/PDMS for beers, and CAR/PDMS for whiskys (extraction time: $60 \mathrm{~min} ; 30 \%$ (w/v) NaCl; $750 \mathrm{rpm}$ ).

phenomenon is controlled from the liquid to the gaseous phase, as well as, the adsorption/absorption onto the fibre coating. The influence of the extraction temperature on the amount of VOCs and SVOCs in wine, beer and whisky, extracted by HS-SPME using the selected fibre for each matrix, i.e. PDMS, DVB/CAR/PDMS and CAR/PDMS, respectively, was investigated by sampling WW1 wine, B1 beer and SW5 whisky, at different temperatures, $30,40,50$ and $60^{\circ} \mathrm{C}$, during $60 \mathrm{~min}$. The results are summarised in Fig. 3. As it can be seen the optimal temperature differs according to the nature of VOCs and SVOCs present in the matrix. For wines VOCs and SVOCs isolation, a significant decrease in the extraction efficiency was observed. As the temperature rises more analytes are released into the headspace, but due to decrease of partition coefficients the absorption of analytes is reduced. The chemical families that are the most affected by the temperature raise are the ethyl esters from fatty acids and higher alcohols. 


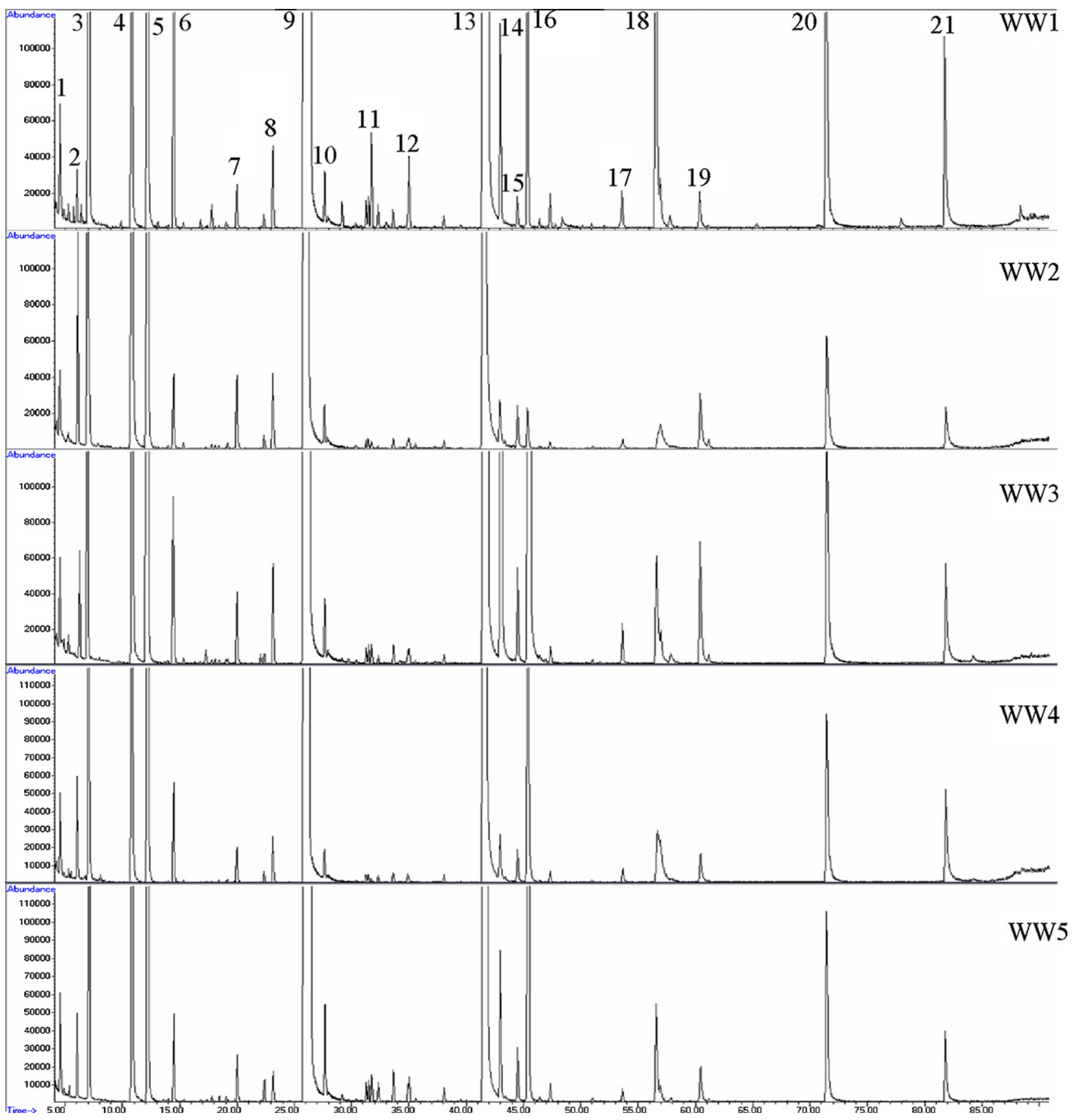

Fig. 4 - Total ion current chromatograms (TIC) of wine VOCs and SVOCs after SPME extraction with PDMS fibre (extraction temperature: $30{ }^{\circ} \mathrm{C}$; extraction time: $60 \mathrm{~min} ; 30 \%$ (w/v) NaCl; stirring: $750 \mathrm{rpm}$ ). Peak identification: (1) ethyl butanoate; (2) 2-methyl-1-propanol; (3) isoamyl acetate; (4) 3-methyl-1-butanol; (5) ethyl hexanoate; (6) hexyl acetate; (7) 1-hexanol; (8) 3-octanol (IS); (9) ethyl octanoate; (10) Isoamyl hexanoate; (11) geraniol acetate; (12) linalool; (13) ethyl decanoate; (14) isoamyl octanoate; (15) ethyl 9-decenoate; (16) 2-phenylethyl acetate; (17) ethyl dodecanoate; (18) $\beta$-phenylethanol; (19) octanoic acid; (20) nonanoic acid; (21) ethyl succinate; (22) decanoic acid.

$30^{\circ} \mathrm{C}$ was selected for VOCs and SVOCs isolation in the studied white wines. As seen in Fig. 3,30 and $40^{\circ} \mathrm{C}$ are the temperatures that enable the best extraction efficiency of VOCs and SVOCs from beers and whiskys, respectively.

Two opposite phenomena take place when the extraction temperature is increased. For the low molecular mass com- pounds, a negative effect was observed at the beginning of the chromatogram at higher extraction temperature. In the meanwhile, at the end of the chromatogram a significant increase in the method sensitivity, with the rise of extraction temperature, was verified. The optimized method conditions are summarised in Table 5. 


\subsection{HS-SPME-GC-qMSD method application to estimate the volatile profile in beverages}

The proposed HS-SPME method, optimized and validated was applied to evaluate and compare the volatile patterns in commercial alcoholic beverages. Each matrix was analysed four times using the best sampling conditions described in Table 5. To confirm the versatility and suitability of the optimized SPME method, it was used to study the volatile fraction composition of three different alcoholic beverages: beer, wine and whisky. The technique used made it possible to identify 44 VOCs and SVOCs in the five white wine samples, 64 VOCs and SVOCs in the five beers and 104 VOCs and SVOCs in the whisky samples. Altogether, only 21 volatiles were simultaneously identified in all five wine samples, 17 in beer samples and 22 in whisky samples.

Most of the volatile compounds were identified by a library search NIST05. In some cases a comparison with authentic compounds was performed. The Kováts retention indices were calculated for each peak and compared with the literature in order to ensure the correct identification of the compounds.

\subsubsection{Wine volatile profile}

A typical GC-qMSD chromatogram obtained from a WW1 wine using the experimental conditions discussed above is shown in Fig. 4. More than 40 volatile compounds belonging to several chemical classes were positively identified, including higher alcohols, fatty acids ethyl esters, fatty acid, acetates, isoamyl esters, terpenoids/ $\mathrm{C}_{13}$-norisoprenoids compounds, carbonyl compounds and miscellaneous. The major fermentation compounds such as ethyl esters, higher alcohols and fatty acids, constitute a main part of the flavour of the young white wines. Table 6 summarises the average peak area $(n=4)$ and the relative composition for the five analysed white wines. The relative composition of every flavour compound was calculated as the percent ratio of the respective peak area relative to the total peak area. From Fig. 4 and Table 6, it is apparent that there were no significant qualitative and quantitative differences between the volatile composition of the studied wines, which can be a consequence of the similarity in the used grape varieties (Table 1) and elaboration/vinification processes of WW1-WW5 wines.

WW5 wines present higher amounts of volatile and semivolatile compounds than WW3 wines, which turn were higher than WW1, WW2 and WW4 wines. WW1 wines are characterised by the presence of terpenoids $/ \mathrm{C}_{13}$-norisoprenoids, fatty acids and higher alcohol acetates, which corroborates with the typical floral and fruity nuances of these wines. WW5 wines are characterised for their high content in higher alcohols, due to the presence of significant quantities of 3methyl-1-butanol and $\beta$-phenylethanol. In contrast, they have the lowest levels of fatty acids. The content of higher alcohols acetates and isoamyl esters found in WW1 wines are higher than those in the other wines. Isoamyl esters are predominant in WW3 wine samples. Fig. 5 presents the distribution of compound classes by wine sample.

The fatty acids ethyl esters are quantitatively the largest group of volatile compounds found in the studied white wines. Ethyl octanoate, ethyl decanoate, ethyl hexanoate, ethyl dodecanoate and ethyl butanoate were dominant. These compounds, namely C4-C10 compounds, make a positive contribution to the general quality of wines being responsible for their "fruity" and "floral" sensory properties. It can also be verified, that the ethyl esters of fatty acids were more abundant than the acetates of higher alcohols.

The second most abundant isolated group were the higher alcohols, to which corresponds $9.3,17.3,12.3,11.4$ and $29.3 \%$ of all volatiles analysed by SPME $\mathrm{PDMS}_{-\mathrm{GC}-\mathrm{qMSD}}$, in WW1, WW2, WW3, WW4, and WW5 wines, respectively. At concentrations above $300 \mathrm{mg} \mathrm{L}^{-1}$, they are regarded as negative quality factors. The main components of this group are 3-methylbutan-1ol, which presence may cause "bitter, harsh, alcohol, fusel", $\beta$-phenylethanol "pollen, roses, floral" notes, and hexan-1-ol, which supply "herbaceous, vegetal" nuances to wine when its concentration surpass their odour threshold values.

Fatty acids have been described with fruity, cheese, fatty and rancid notes. Among these compounds, octanoic acid and decanoic acid were present at higher contents in the five wines analysed. Their mean values were very similar and they did not present significant differences (Fig. 5). The highest content was observed for octanoic acid. Hexanoic acid and 3-methylbutanoic acid were detected only in WW1, WW3, WW5 and WW2 and WW5, respectively. Although the presence of C6-C10 fatty acids is usually related to the appearance of negative odours, they are very important for the aromatic equilibrium in wines because they are opposed to the hydrolysis of the corresponding esters.

The terpenoids content of a wine is considered to be a quality factor. These wine constituents are fragrant and are no doubt important to the general enhancement of floral and fruity aromas. These compounds have been reported as having a determinant role in the wine aroma profile due to their very pleasant aroma and very low olfactory thresholds, so that they can be perceived during wine tasting even in low concentrations, due as well, to several synergic and antagonist effects observed between them. This group showed the lowest values in the studied wines. Using dynamic headspace SPME only $0.2 \%$ (WW2 and WW4) to $1.4 \%$ (WW1) were found. Many of the terpenoids and $\mathrm{C}_{13}$-norisoprenoids identified in this study are typical constituents of different wines. Thus linalool, terpinolene and geraniol, have been reported previously as constituents of wines from Vitis vinifera $L$. varieties. The major compounds of this chemical family found in white

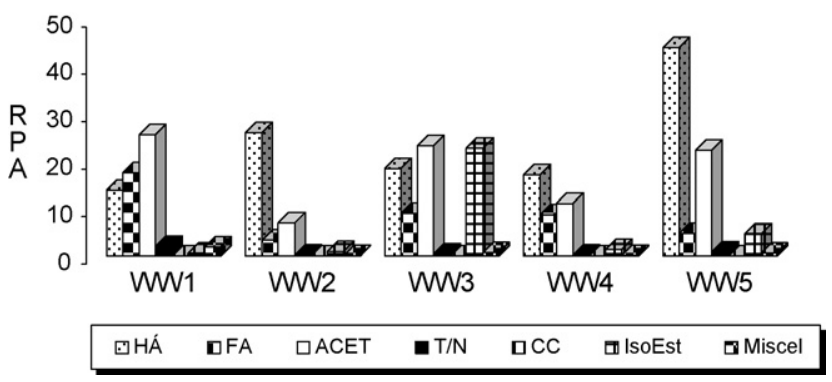

Fig. 5 - Profile of the major volatile families found in the analysed wines (HA: higher alcohols; FA: fatty acids; ACET: higher alcohol acetates; T/N: terpenopid and $\mathrm{C}_{13}$-norisoprenoids; CC: carbonyl compounds; IsoEst: isoamyl esters; Miscel: miscellaneous). 
Table 6 - VOCs and SVOCs identified and quantified (RPA, \%) in different white wine samples after dynamic HS-SPME extraction using PDMS coating and GC-qMSD analysis (extraction temperature: $30^{\circ} \mathrm{C}$; extraction time: $60 \mathrm{~min}$, sample with $30 \%, \mathrm{w} / \mathrm{v}, \mathrm{NaCl}$ )

\begin{tabular}{|c|c|c|c|c|c|c|c|c|c|c|c|c|c|}
\hline \multirow[t]{2}{*}{$\mathrm{RT}(\min )$} & \multirow[t]{2}{*}{$\mathrm{KI}^{\mathrm{a}}$} & \multirow[t]{2}{*}{ Compounds } & \multirow[t]{2}{*}{ Identity $^{\mathrm{b}}$} & \multicolumn{2}{|c|}{ WW1 } & \multicolumn{2}{|c|}{ WW2 } & \multicolumn{2}{|c|}{ WW3 } & \multicolumn{2}{|c|}{ WW4 } & \multicolumn{2}{|c|}{ WW5 } \\
\hline & & & & $\begin{array}{c}\text { Average } \\
\text { peak area }\end{array}$ & $\mathrm{RPA}^{\mathrm{c}}(\%)$ & $\begin{array}{l}\text { Average } \\
\text { Peak area }\end{array}$ & RPA (\%) & $\begin{array}{c}\text { Average } \\
\text { peak area }\end{array}$ & RPA (\%) & $\begin{array}{c}\text { Average } \\
\text { peak area }\end{array}$ & RPA (\%) & $\begin{array}{c}\text { Average } \\
\text { peak area }\end{array}$ & RPA (\%) \\
\hline 4.521 & 1078 & Ethyl butyrate & A, B & $3.46 \times 10^{6}$ & 0.16 & $2.50 \times 10^{6}$ & 0.19 & $1.78 \times 10^{6}$ & 0.08 & $2.19 \times 10^{6}$ & 0.23 & $3.30 \times 10^{6}$ & 0.12 \\
\hline 4.927 & 1097 & Ethyl methanoate & B & $-d$ & - & - & - & $2.37 \times 10^{5}$ & 0.01 & - & - & - & - \\
\hline 5.342 & 1114 & Ethyl isovalerate & A, B & $3.39 \times 10^{5}$ & 0.02 & - & - & $3.07 \times 10^{5}$ & 0.01 & $2.74 \times 10^{5}$ & 0.03 & $3.47 \times 10^{5}$ & 0.01 \\
\hline 6.368 & 1147 & 2-Methyl-1-propanol & A, B & $2.88 \times 10^{7}$ & 0.13 & $2.46 \times 10^{6}$ & 1.50 & $1.88 \times 10^{6}$ & 0.08 & $4.50 \times 10^{6}$ & 0.46 & $1.07 \times 10^{6}$ & 0.04 \\
\hline 6.907 & 1167 & Isoamyl acetate & B & $2.56 \times 10^{7}$ & 1.18 & $2.00 \times 10^{7}$ & 2.60 & $2.49 \times 10^{7}$ & 1.05 & $1.57 \times 10^{7}$ & 1.62 & $5.62 \times 10^{7}$ & 2.12 \\
\hline 9.902 & 1246 & Limonene & A, B & - & - & - & - & - & - & - & - & $1.89 \times 10^{6}$ & 0.01 \\
\hline 10.804 & 1267 & 3-Methyl-1-butanol & A, B & $5.91 \times 10^{7}$ & 2.69 & $3.50 \times 10^{7}$ & 3.98 & $5.04 \times 10^{7}$ & 1.91 & $6.47 \times 10^{7}$ & 6.61 & $3.41 \times 10^{7}$ & 1.30 \\
\hline 12.104 & 1293 & Ethyl hexanoate & A, B & $1.10 \times 10^{8}$ & 5.10 & $4.40 \times 10^{6}$ & 0.33 & $7.26 \times 10^{7}$ & 3.07 & $4.68 \times 10^{7}$ & 4.89 & $1.03 \times 10^{8}$ & 3.89 \\
\hline 13.190 & 1316 & Styrene & A, B & - & - & - & - & - & - & - & - & $1.76 \times 10^{5}$ & 0.01 \\
\hline 14.389 & 1340 & Hexyl acetate & A, B & $3.63 \times 10^{6}$ & 0.17 & $3.63 \times 10^{6}$ & 0.14 & $6.49 \times 10^{6}$ & 0.28 & $2.62 \times 10^{6}$ & 0.27 & $1.27 \times 10^{7}$ & 0.48 \\
\hline 17.789 & 1400 & Linalool & A, B & - & - & - & - & - & - & - & - & $8.76 \times 10^{5}$ & 0.03 \\
\hline 18.462 & 1413 & Ethyl heptanoate & A, B & $2.19 \times 10^{5}$ & 0.01 & - & 0.04 & - & - & - & - & - & - \\
\hline 20.021 & 1443 & 1-Hexanol & A, B & $2.46 \times 10^{6}$ & 0.11 & $1.87 \times 10^{6}$ & 0.18 & $1.84 \times 10^{6}$ & 0.08 & $2.55 \times 10^{6}$ & 0.26 & $1.46 \times 10^{6}$ & 0.05 \\
\hline 22.123 & 1480 & 3-Hexen-1-ol & A, B & - & - & - & - & $1.53 \times 10^{5}$ & 0.01 & - & - & - & - \\
\hline 22.384 & 1485 & Methyl octanoate & A, B & $9,97 \times 10^{5}$ & 0.05 & $5.31 \times 10^{5}$ & 59.42 & $3.01 \times 10^{5}$ & 0.01 & $4.83 \times 10^{5}$ & 0.05 & $4.64 \times 10^{6}$ & 0.02 \\
\hline 25.872 & 1547 & Ethyl octanoate & B & $1.62 \times 10^{9}$ & 77.27 & $7.81 \times 10^{8}$ & 0.02 & $1.10 \times 10^{9}$ & 46.89 & $6.25 \times 10^{8}$ & 60.41 & $1.33 \times 10^{9}$ & 50.05 \\
\hline 27.644 & 1577 & Isopentyl hexanoate & A, B & $3.72 \times 10^{6}$ & 0.18 & $1.13 \times 10^{6}$ & 0.03 & $1.99 \times 10^{5}$ & 0.09 & $1.35 \times 10^{6}$ & 0.13 & $1.77 \times 10^{6}$ & 0.07 \\
\hline 27.863 & 1580 & Acetic acid & B & $2.83 \times 10^{5}$ & 0.01 & - & - & $9.86 \times 10^{4}$ & 0.00 & $2.09 \times 10^{5}$ & 0.02 & $2.67 \times 10^{5}$ & 0.01 \\
\hline 30.334 & 1619 & $\mathrm{HTHF}^{\mathrm{e}}$ & B & - & - & - & - & - & - & - & - & $1.78 \times 10^{5}$ & 0.01 \\
\hline 31.26 & 1632 & $\alpha$-Ionone & B & $8,24 \times 10^{5}$ & 0.04 & $3.02 \times 10^{5}$ & 0.03 & $4.37 \times 10^{5}$ & 0.02 & $2.02 \times 10^{5}$ & 0.02 & $7.95 \times 10^{5}$ & 0.03 \\
\hline 31.471 & 1635 & Terpinolene & A, B & $9,21 \times 10^{5}$ & 0.04 & $3.48 \times 10^{5}$ & 0.01 & $5.87 \times 10^{5}$ & 0.03 & - & - & $9.64 \times 10^{5}$ & 0.04 \\
\hline 31.739 & 1639 & Geraniol & $\mathrm{A}, \mathrm{B}$ & $1.19 \times 10^{6}$ & 0.06 & - & 0.02 & - & - & - & - & $3.37 \times 10^{6}$ & 0.13 \\
\hline 32.308 & 1647 & Propyl octanoate & A, B & $8.68 \times 10^{5}$ & 0.04 & $3.32 \times 10^{5}$ & 26.45 & - & - & $3.0510^{5}$ & 0.03 & $8.79 \times 10^{5}$ & 0.03 \\
\hline 33.625 & 1665 & Ethyl nonanoate & B & $1.40 \times 10^{6}$ & 0.07 & $4.39 \times 10^{5}$ & 0.17 & $6.6010^{5}$ & 0.03 & $3.8710^{5}$ & 0.04 & $6.42 \times 10^{5}$ & 0.02 \\
\hline 34.818 & 1806 & Isobutyl octanoate & B & $6.54 \times 10^{5}$ & 0.03 & - & 0.12 & - & - & - & - & - & - \\
\hline 35.018 & 1810 & $\beta$-Myrcene & B & $1.11 \times 10^{6}$ & 0.05 & $1.82 \times 10^{5}$ & 1.91 & $4.3610^{5}$ & 0.02 & $2.8610^{5}$ & 0.04 & $2.80 \times 10^{6}$ & 0.11 \\
\hline 35.554 & 1821 & Octan-1-ol & B & $1.91 \times 10^{5}$ & 0.01 & - & 0.04 & - & - & - & - & - & - \\
\hline 38.028 & 1868 & 2-Methyl decanoic acid & B & - & - & $2.02 \times 10^{5}$ & 0.06 & - & - & - & - & $3.97 \times 10^{5}$ & 0.01 \\
\hline 41.49 & 1935 & Ethyl decanoate & B & $2.99 \times 10^{8}$ & 10.72 & $3.46 \times 10^{8}$ & 0.75 & $6.42 \times 10^{8}$ & 30.37 & $2.46 \times 10^{8}$ & 22.37 & $9.90 \times 10^{8}$ & 37.51 \\
\hline 43.013 & 1965 & 3-Methyl octanoate & $\mathrm{A}, \mathrm{B}$ & $3.05 \times 10^{6}$ & 0.11 & $2.22 \times 10^{6}$ & 0.17 & $6.40 \times 10^{7}$ & 2.74 & $2.12 \times 10^{6}$ & 0.20 & - & - \\
\hline 44.533 & 1993 & Diethyl succinate & A, B & $1.60 \times 10^{6}$ & 0.06 & $1.62 \times 10^{6}$ & 1.01 & $3.03 \times 10^{6}$ & 0.13 & $1.77 \times 10^{6}$ & 0.18 & $1.20 \times 10^{6}$ & 0.05 \\
\hline 45.356 & 2010 & Ethyl 9-decenoate & B & $3.13 \times 10^{7}$ & 1.13 & $2.51 \times 10^{7}$ & 0.56 & $2.49 \times 10^{8}$ & 10.73 & $1.23 \times 10^{6}$ & 0.17 & $1.62 \times 10^{7}$ & 0.61 \\
\hline 47.341 & 2049 & $\operatorname{TDN}^{f}$ & A, B & $4.78 \times 10^{5}$ & 0.02 & $5.20 \times 10^{5}$ & 0.19 & $6.50 \times 10^{5}$ & 0.03 & - & - & $1.40 \times 10^{6}$ & 0.05 \\
\hline 51.047 & 2124 & 2,3-Dihydrofuran & A, B & $1.78 \times 10^{5}$ & 0.01 & - & 0.18 & - & - & $1.61 \times 10^{6}$ & 0.03 & - & - \\
\hline 53.668 & 2182 & 2-Phenylethyl acetate & B & $4.48 \times 10^{5}$ & 0.02 & $7.69 \times 10^{5}$ & 1.50 & $1.61 \times 10^{6}$ & 0.07 & $1.19 \times 10^{7}$ & 0.03 & $1.74 \times 10^{6}$ & 0.07 \\
\hline 56.601 & 2246 & Ethyl dodecanoate & B & $2.9210^{6}$ & 0.10 & $1.0110^{6}$ & 2.60 & $1.19 \times 10^{7}$ & 0.64 & $3.57 \times 10^{6}$ & 0.24 & $2.17 \times 10^{7}$ & 0.73 \\
\hline 56.989 & 2255 & Hexanoic acid & B & $7.47 \times 10^{5}$ & 0.03 & - & 3.98 & $1.69 \times 10^{6}$ & 0.08 & - & - & $2.64 \times 10^{6}$ & 0.10 \\
\hline 57.821 & 2273 & 3-Methyl pentanoate & A, B & - & - & - & - & $9.39 \times 10^{5}$ & 0.05 & - & - & - & - \\
\hline 60.454 & 2328 & $\tilde{\beta}$-Phenylethanol & B & $1.69 \times 10^{6}$ & 0.06 & $1.92 \times 10^{6}$ & 0.33 & $5.57 \times 10^{6}$ & 0.24 & $3.42 \times 10^{6}$ & 0.35 & $1.92 \times 10^{6}$ & 0.07 \\
\hline 61.183 & 2343 & TBMPg & B & - & - & - & - & $2.9910^{6}$ & 0.01 & $2.9910^{5}$ & 0.02 & - & - \\
\hline 71.463 & 2524 & Octanoic acid & A, B & $6.81 \times 10^{6}$ & 0.24 & $1.31 \times 10^{7}$ & 0.14 & $1.7910^{6}$ & 0.77 & $7.6410^{6}$ & 0.74 & $3.01 \times 10^{7}$ & 1.14 \\
\hline
\end{tabular}




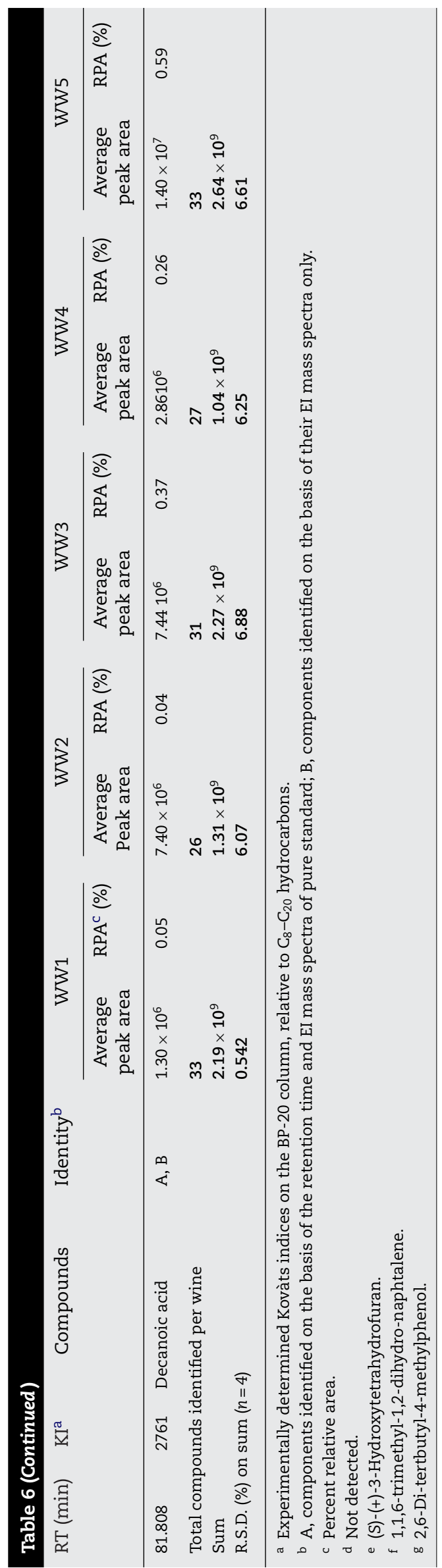

wines were 1,1,6-trimethyl-1,2-dihydronaphtalene (TDN) and $\beta$-damascenone. The presence of $\mathrm{C}_{13}$-norisoprenoids, TDN and $\beta$-damascenone, is also considered to be a quality factor, as they supply an agreeable scent of flowers, fruits, tea, honey-like, black currant or cassis notes, except for TDN that exhibits a kerosene-like odour. They are present in free and odourless glycosidically bound forms in grapes and wines, and can be released liberated by acid catalysed hydrolysis during conservation.

\subsubsection{Beer volatile profile}

As shown in Table 7, a total of 64 volatile and semivolatile organic compounds belonging to several chemical classes were positively identified in the five beer samples. Fifteen of these volatiles: 2-methyl-1-propanol, isoamyl acetate, 3-methyl-1-butanol, ethyl hexanoate, heptyl acetate, ethyl octanoate, octyl acetate, linalool, ethyl decanoate, 3methyl butanoic acid, 2-phenylethyl acetate, $\beta$-phenylethanol, octanoic acid and decanoic acid, were detected in all samples (Table 7) but the ratio between the single components were different for each beer. Some of these 15 compounds, such as isoamyl acetate, hepty acetate, octyl acetate, linalool and $\beta$-phenylethanol, have been reported as common components of different beer types [22,23]. A characteristic GC-qMSD profile of each beer obtained with DVB/CAR/PDMS using the experimental conditions discussed above is shown in Fig. 6.

Table 7 summarises the average $(n=4)$ relative composition determined in investigated beers. The relative composition of every flavour compound was calculated as the percent ratio of the respective peak area relative to the total peak area (RPA, \%). Fig. 7 presents the distribution of compound classes by beer sample. As shown Fig. 8, ethyl esters followed by higher alcohols were the most abundant compound classes. Ethyl esters are characterised by their fruity flavour and plays an important role in the overall flavour balance. The main esters of the HS-SPMS $\mathrm{DVB}_{\mathrm{CAR} / \mathrm{PDMS}}$ samples of beer were ethyl octanoate, ethyl decanoate, ethyl hexanoate, ethyl dodecanoate and 2phenylethyl acetate.

Alcohols contribute to the strong and pungent smell and taste of beer; only ethanol, 2-methyl-butan-1-ol, 3methylbutan-1-ol, and octan-1-ol were identified in the five beer samples. The higher alcohols (i.e. of higher molecular weight than ethanol) are important as the immediate precursors of the more flavour-active esters, so that higher alcohol formation needs regulation to ensure that, in turn, ester production is controlled.

Acids constitute an important group of aroma compounds that can contribute with fruity, cheesy and fatty odours to beer's sensory properties. They also contribute to bitterness, adstringency and rancidity. Four from the five acids identified in the five investigated beers: 3-methylbutanoic acid, and C6, C8 and C10 fatty acids were detected in all beer samples in significantly different amounts.

Each beer type showed a typical composition. B1 beer (Fig. 6) is characterised by a high amount of ethyl octanoate, ethyl decanoate, 3-methyl-1-butanol and ethyl hexanoate. Some identified compounds are specific to this sample. Therefore, 3-heptene and 5-hydroxymethylfurfural were identified only in B1 beer sample. This sample is also characterized by the absence of (E)-2-nonen-1-ol, present in B2-B5 samples. 
Table 7 - Compounds identified and quantified (RPA, \%) in beer samples after dynamic HS-SPME $30^{\circ} \mathrm{C}$; extraction time: $60 \mathrm{~min}$, sample with $30 \%$, w/v NaC

\begin{tabular}{|c|c|c|c|c|c|c|c|c|c|c|c|c|c|}
\hline \multirow[t]{2}{*}{ RT (min) } & \multirow[t]{2}{*}{$\mathrm{KI}^{\mathrm{a}}$} & \multirow[t]{2}{*}{ Compound } & \multirow[t]{2}{*}{ Identity $^{b}$} & \multicolumn{2}{|l|}{ B1 } & \multicolumn{2}{|c|}{ B2 } & \multicolumn{2}{|c|}{ B3 } & \multicolumn{2}{|c|}{ B4 } & \multicolumn{2}{|c|}{ B5 } \\
\hline & & & & Average peak area & $\operatorname{RPA}^{\mathrm{c}}(\%)$ & $\begin{array}{c}\text { Average } \\
\text { Peak area }\end{array}$ & RPA (\%) & $\begin{array}{l}\text { Average } \\
\text { peak area }\end{array}$ & RPA (\%) & $\begin{array}{l}\text { Average } \\
\text { peak area }\end{array}$ & RPA (\%) & $\begin{array}{c}\text { Average } \\
\text { Peak area }\end{array}$ & RPA (\%) \\
\hline 4.479 & 1075 & Ethyl butanoate & $\mathrm{A}, \mathrm{B}$ & $4.58 \times 10^{6}$ & 0.46 & - & - & $2.59 \times 10^{6}$ & 0.35 & $5.50 \times 10^{6}$ & 1.11 & $4.24 \times 10^{6}$ & 0.58 \\
\hline 4.586 & 1080 & Toluene & A, B & $-^{\mathrm{d}}$ & - & $1.35 \times 10^{6}$ & 0.66 & - & - & - & - & - & - \\
\hline 6.335 & 1149 & 2-Methyl-1-propanol & A, B & $3.65 \times 10^{5}$ & 0.37 & $6.31 \times 10^{5}$ & 0.30 & $3.31 \times 10^{6}$ & 0.46 & $4.49 \times 10^{6}$ & 0.91 & $3.45 \times 10^{6}$ & 0.48 \\
\hline 6.872 & 1165 & Isoamyl acetate & A, B & $1.32 \times 10^{7}$ & 13.29 & $1.69 \times 10^{7}$ & 8.19 & $9.83 \times 10^{7}$ & 13.31 & $9.89 \times 10^{7}$ & 20.00 & $1.16 \times 10^{8}$ & 15.96 \\
\hline 7.470 & 1183 & $p$-Xylene & A, B & - & - & $5.86 \times 10^{5}$ & 0.28 & - & - & - & - & - & - \\
\hline 7.813 & 1192 & 1-Butanol & A, B & - & - & - & - & - & - & $4.19 \times 10^{5}$ & 0.09 & $2.02 \times 10^{5}$ & 0.03 \\
\hline 8.120 & 1200 & Butanal & A, B & - & - & $8.97 \times 10^{4}$ & 0.04 & - & - & - & - & - & - \\
\hline 8.383 & 1207 & $\beta$-Myrcene & B & - & - & - & - & $2.15 \times 10^{5}$ & 0.03 & - & - & $1.80 \times 10^{5}$ & 0.02 \\
\hline 9.518 & 1233 & 2-Ethylhexanal & B & - & - & $6.60 \times 10^{5}$ & 0.32 & - & - & - & - & - & - \\
\hline 9.575 & 1234 & $\mathrm{BMECB}^{\mathrm{e}}$ & B & $1.72 \times 10^{5}$ & 0.02 & - & - & - & - & - & - & - & - \\
\hline 9.794 & 1239 & Pentyl propenoate & B & - & - & - & - & - & - & - & - & $3.02 \times 10^{5}$ & 0.04 \\
\hline 9.800 & 1239 & Limonene & A, B & - & - & $7.15 \times 10^{4}$ & 0.04 & $1.21 \times 10^{5}$ & 0.02 & - & - & - & - \\
\hline 10.889 & 1268 & 3-Methyl-1-butanol & A, B & $8.04 \times 10^{7}$ & 8.06 & $2.42 \times 10^{7}$ & 11.59 & $6.72 \times 10^{7}$ & 9.28 & $9.75 \times 10^{7}$ & 19.77 & $7.61 \times 10^{7}$ & 10.49 \\
\hline 12.098 & 1293 & Ethyl hexanoate & A, B & $6.55 \times 10^{7}$ & 6.57 & $6.92 \times 10^{6}$ & 3.39 & $4.14 \times 10^{7}$ & 5.60 & $4.59 \times 10^{7}$ & 9.28 & $3.66 \times 10^{7}$ & 5.00 \\
\hline 13.154 & 1315 & 3-Octanone & B & - & - & $2.39 \times 10^{5}$ & 0.10 & - & - & - & - & - & - \\
\hline 13.763 & 1328 & $\beta$-Cymene & B & - & - & $1.44 \times 10^{5}$ & 0.07 & - & - & - & - & - & - \\
\hline 14.389 & 1340 & Hexyl acetate & A, B & $1.15 \times 10^{6}$ & 0.12 & $5.47 \times 10^{5}$ & 0.27 & - & - & $1.07 \times 10^{6}$ & 0.22 & $9.56 \times 10^{5}$ & 0.13 \\
\hline 15.283 & 1357 & (Z)-2-Hexene & B & - & - & $3.27 \times 10^{5}$ & 0.14 & - & - & - & - & $5.23 \times 10^{5}$ & 0.07 \\
\hline 17.384 & 1393 & Ethyl 3-hexenoate & B & - & - & - & - & - & - & $1.48 \times 10^{5}$ & 0.03 & - & - \\
\hline 18.137 & 1407 & 2-Methylfuran & B & - & - & $3.26 \times 10^{5}$ & 0.16 & - & - & - & - & - & - \\
\hline 18.374 & 1412 & Ethyl heptanoate & A, B & $9.17 \times 10^{5}$ & 0.09 & - & - & $7.94 \times 10^{5}$ & 0.11 & - & - & $2.49 \times 10^{5}$ & 0.03 \\
\hline 19.996 & 1442 & 2-Propenal & B & - & - & - & - & - & - & $2.09 \times 10^{5}$ & 0.03 & $8.73 \times 10^{4}$ & 0.01 \\
\hline 20.010 & 1443 & 4-Methyl-1-pentanol & A, B & - & - & $1.24 \times 10^{5}$ & 0.06 & - & - & - & - & - & - \\
\hline 21.283 & 1466 & Heptyl acetate & A, B & $3.54 \times 10^{6}$ & 0.36 & $7.83 \times 10^{5}$ & 0.39 & $5.23 \times 10^{6}$ & 0.71 & $4.98 \times 10^{5}$ & 0.10 & $1.02 \times 10^{6}$ & 0.14 \\
\hline 22.440 & 1486 & (E)-2-Nonen-1-ol & B & - & - & $1.21 \times 10^{6}$ & 0.48 & $8.10 \times 10^{5}$ & 0.12 & $8.42 \times 10^{5}$ & 0.17 & $8.99 \times 10^{5}$ & 0.12 \\
\hline 22.424 & 1486 & 3-Heptene & A, B & $1.37 \times 10^{5}$ & 0.01 & - & - & - & - & - & - & - & - \\
\hline 25.669 & 1543 & Ethyl octanoate & B & $4.36 \times 10^{8}$ & 43.73 & $1.57 \times 10^{7}$ & 9.00 & $2.69 \times 10^{8}$ & 35.50 & $5.41 \times 10^{7}$ & 10.92 & $2.48 \times 10^{8}$ & 33.22 \\
\hline 26.369 & 1555 & Isomenthone & B & - & - & - & - & - & - & $7.10 \times 10^{5}$ & 0.14 & - & - \\
\hline 27.582 & 1576 & 1-Heptanol & B & - & - & - & - & $2.81 \times 10^{5}$ & 0.04 & $2.01 \times 10^{5}$ & 0.04 & $3.00 \times 10^{5}$ & 0.04 \\
\hline 27.611 & 1576 & 3-Methylhepthyl acetate & B & - & - & - & - & - & - & - & - & $7.72 \times 10^{5}$ & 0.13 \\
\hline 27.810 & 1579 & Guaiacol & A, B & $1.05 \times 10^{6}$ & 0.11 & - & - & $1.46 \times 10^{6}$ & 0.23 & $1.85 \times 10^{6}$ & 0.41 & $2.86 \times 10^{6}$ & 0.38 \\
\hline 27.643 & 1577 & 3-Methyl-1-hexanol & B & - & - & $4.46 \times 10^{5}$ & 0.22 & - & - & - & - & - & - \\
\hline 27.892 & 1530 & Acetic acid & A, B & - & - & $8.02 \times 10^{5}$ & 0.39 & - & - & - & - & - & - \\
\hline 28.363 & 1588 & Isomenthone & B & - & - & & - & - & - & $2.31 \times 10^{5}$ & 0.05 & - & - \\
\hline 28.951 & 1598 & Octyl acetate & B & $4.40 \times 10^{6}$ & 0.44 & $4.38 \times 10^{7}$ & 0.22 & $3.78 \times 10^{6}$ & 0.50 & $5.34 \times 10^{5}$ & 0.11 & $2.13 \times 10^{6}$ & 0.28 \\
\hline 30.376 & 1619 & 2-Ethyl-1-hexanol & B & $2.56 \times 10^{6}$ & 0.26 & $1.20 \times 10^{6}$ & 5.89 & $3.15 \times 10^{6}$ & 0.44 & - & - & - & - \\
\hline 30.403 & 1620 & Decanal & B & - & - & - & - & - & - & $1.81 \times 10^{6}$ & 0.23 & $1.02 \times 10^{5}$ & 0.01 \\
\hline 33.469 & 1663 & 4-Cyclohexylresorcinol & A, B & $8.14 \times 10^{4}$ & 0.01 & - & - & $1.23 \times 10^{5}$ & 0.02 & - & - & $4.78 \times 10^{5}$ & 0.06 \\
\hline 33.596 & 1664 & Ethyl nonanoate & A, B & $6.52 \times 10^{5}$ & 0.07 & - & - & $5.29 \times 10^{5}$ & 0.05 & - & - & $8.92 \times 10^{5}$ & 0.12 \\
\hline 35.001 & 1683 & Linalool & A, B & $5.15 \times 10^{5}$ & 0.05 & $1.02 \times 10^{6}$ & 0.50 & $1.80 \times 10^{5}$ & 0.03 & $1.14 \times 10^{6}$ & 0.23 & $1.59 \times 10^{6}$ & 0.22 \\
\hline 35.538 & 1690 & 1-Octanol & A, B & - & - & - & - & $1.25 \times 10^{6}$ & 0.18 & $1.82 \times 10^{6}$ & 0.37 & - & - \\
\hline 35.586 & 1691 & 1,2-Diethyl-cyclobutane & B & $1.01 \times 10^{6}$ & 0.10 & $4.34 \times 10^{5}$ & 0.22 & - & - & - & - & $2.43 \times 10^{5}$ & 0.03 \\
\hline
\end{tabular}




\section{Table 7 (Continued)}

\begin{tabular}{|c|c|c|c|c|c|c|c|c|c|c|c|c|c|}
\hline \multirow[t]{2}{*}{$\mathrm{RT}$ (min) } & \multirow[t]{2}{*}{$\mathrm{KI}^{\mathrm{a}}$} & \multirow[t]{2}{*}{ Compound } & \multirow[t]{2}{*}{ Identity $^{\mathrm{b}}$} & \multicolumn{2}{|l|}{ B1 } & \multicolumn{2}{|c|}{ B2 } & \multicolumn{2}{|c|}{ B3 } & \multicolumn{2}{|c|}{ B4 } & \multicolumn{2}{|c|}{ B5 } \\
\hline & & & & Average peak area & $\operatorname{RPA}^{\mathrm{c}}(\%)$ & $\begin{array}{c}\text { Average } \\
\text { Peak area }\end{array}$ & RPA (\%) & $\begin{array}{c}\text { Average } \\
\text { peak area }\end{array}$ & RPA (\%) & $\begin{array}{c}\text { Average } \\
\text { peak area }\end{array}$ & RPA (\%) & $\begin{array}{c}\text { Average } \\
\text { Peak area }\end{array}$ & RPA (\%) \\
\hline 41.269 & 1930 & Ethyl decanoate & B & $9.11 \times 10^{7}$ & 9.10 & $3.73 \times 10^{5}$ & 0.15 & $6.87 \times 10^{7}$ & 9.22 & $9.37 \times 10^{6}$ & 1.89 & $4.86 \times 10^{7}$ & 6.37 \\
\hline 42.424 & 1953 & Ethyl benzoate & B & $1.59 \times 10^{5}$ & 0.02 & - & - & $1.59 \times 10^{5}$ & 0.02 & - & - & - & - \\
\hline 42.899 & 1963 & Isopentyl hexanoate & B & - & - & - & - & $2.90 \times 10^{5}$ & 0.04 & - & - & - & - \\
\hline 43.14 & 1967 & Citronellyl butyrate & B & $8.63 \times 10^{5}$ & 0.09 & - & - & $6.07 \times 10^{5}$ & 0.08 & - & - & $9.33 \times 10^{5}$ & 0.12 \\
\hline 43.484 & 1974 & 2-Furanmethanol & B & $6.66 \times 10^{5}$ & 0.07 & - & - & $6.02 \times 10^{5}$ & 0.08 & $3.43 \times 10^{5}$ & 0.07 & $2.85 \times 10^{5}$ & 0.06 \\
\hline 43.506 & 1974 & 3-Methyl-2(5H)-furanone & B & - & - & $3.36 \times 10^{5}$ & 0.19 & - & - & - & - & - & - \\
\hline 44.167 & 1987 & 3-Methyl pentanoic acid & A, B & $4.09 \times 10^{5}$ & 0.04 & $1.77 \times 10^{5}$ & 0.09 & $2.89 \times 10^{5}$ & 0.04 & $4.14 \times 10^{5}$ & 0.08 & $1.29 \times 10^{5}$ & 0.04 \\
\hline 44.631 & 1995 & 3-Methyl-1-hexanol & A, B & - & - & - & - & - & - & - & - & $1.29 \times 10^{5}$ & 0.02 \\
\hline 44.647 & 1996 & Decyl acetate & A, B & $4.77 \times 10^{5}$ & 0.05 & - & - & $5.32 \times 10^{5}$ & 0.07 & - & - & - & - \\
\hline 45.415 & 2011 & Ethyl 9-decenoate & B & $1.67 \times 10^{7}$ & 1.67 & - & - & $9.90 \times 10^{6}$ & 1.33 & $6.03 \times 10^{5}$ & 0.12 & $6.05 \times 10^{6}$ & 0.79 \\
\hline 48.723 & 2076 & Cadinene & B & - & - & - & - & - & - & - & - & $1.14 \times 10^{5}$ & 0.03 \\
\hline 50.995 & 2123 & 1-Decanol & $\mathrm{A}, \mathrm{B}$ & $9.64 \times 10^{5}$ & 0.10 & - & - & $1.14 \times 10^{6}$ & 0.16 & $3.23 \times 10^{5}$ & 0.07 & $7.03 \times 10^{5}$ & 0.09 \\
\hline 51.255 & 2129 & 1,7-Heptanediol & B & - & - & - & - & - & - & - & - & $3.11 \times 10^{5}$ & 0.04 \\
\hline 53.679 & 2182 & 2-Phenyl ethyl acetate & A, B & $3.62 \times 10^{7}$ & 3.63 & $3.74 \times 10^{6}$ & 2.13 & $3.79 \times 10^{7}$ & 5.50 & $4.35 \times 10^{7}$ & 8.85 & $7.32 \times 10^{7}$ & 10.34 \\
\hline 56.565 & 2245 & Ethyl dodecanoate & B & $1.98 \times 10^{6}$ & 0.20 & - & - & $1.37 \times 10^{5}$ & 0.02 & $2.80 \times 10^{5}$ & 0.06 & $2.12 \times 10^{5}$ & 0.02 \\
\hline 57.025 & 2255 & Hexanoic acid & B & $3.98 \times 10^{6}$ & 0.40 & $1.14 \times 10^{6}$ & 0.68 & $3.47 \times 10^{6}$ & 0.51 & $2.45 \times 10^{6}$ & 0.50 & $2.71 \times 10^{6}$ & 0.37 \\
\hline 60.438 & 2327 & Phenylethyl alcohol & B & $4.09 \times 10^{7}$ & 4.11 & $8.24 \times 10^{6}$ & 4.13 & $4.95 \times 10^{7}$ & 7.22 & $8.01 \times 10^{7}$ & 16.26 & $6.97 \times 10^{7}$ & 9.47 \\
\hline 63.821 & 2395 & Phenylethyl isobutyrate & B & $4.91 \times 10^{5}$ & 0.05 & - & - & $4.70 \times 10^{5}$ & 0.04 & - & - & - & - \\
\hline 71.569 & 2525 & Octanoic acid & A, B & $4.24 \times 10^{7}$ & 4.26 & $8.93 \times 10^{6}$ & 5.53 & $3.43 \times 10^{7}$ & 5.16 & $1.49 \times 10^{7}$ & 3.06 & $2.14 \times 10^{7}$ & 2.79 \\
\hline 78.259 & 2587 & 2-Methoxy-4-vinylphenol & A, B & $2.39 \times 10^{5}$ & 0.02 & - & - & - & - & - & - & $2.61 \times 10^{5}$ & 0.04 \\
\hline 81.772 & 2761 & Decanoic acid & B & $7.17 \times 10^{6}$ & 0.72 & $2.84 \times 10^{6}$ & 1.95 & $6.40 \times 10^{6}$ & 0.91 & $3.86 \times 10^{6}$ & 0.79 & $3.66 \times 10^{6}$ & 0.43 \\
\hline 88.545 & 2283 & 5-Hydroxymethylfurfural & B & $3.60 \times 10^{5}$ & 0.04 & - & - & - & - & - & - & - & - \\
\hline \multicolumn{4}{|c|}{ Total compounds identified per beer } & \multicolumn{2}{|l|}{35} & \multicolumn{2}{|l|}{32} & \multicolumn{2}{|l|}{35} & \multicolumn{2}{|l|}{31} & \multicolumn{2}{|l|}{40} \\
\hline \multicolumn{4}{|c|}{ Sum } & \multicolumn{2}{|l|}{$1.99 \times 10^{9}$} & \multicolumn{2}{|l|}{$1.99 \times 10^{8}$} & \multicolumn{2}{|l|}{$7.36 \times 10^{8}$} & \multicolumn{2}{|l|}{$4.93 \times 10^{8}$} & \multicolumn{2}{|l|}{$7.50 \times 10^{8}$} \\
\hline \multicolumn{4}{|c|}{ R.S.D. $(\%)$ on $\operatorname{sum}(n=4)$} & \multicolumn{2}{|l|}{10.2} & \multicolumn{2}{|l|}{11.0} & \multicolumn{2}{|l|}{6.59} & \multicolumn{2}{|l|}{9.32} & \multicolumn{2}{|l|}{12.34} \\
\hline
\end{tabular}

a Experimentally determined Kovàts indices on the BP-20 column, relative to $\mathrm{C}_{8}-\mathrm{C}_{20}$ hydrocarbons.

b A, components identified on the basis of the retention time and EI mass spectra of pure standard; B, components identified on the basis of their EI mass spectra only.

c Percent relative area.

d Not detected.

e Trans-1,2-bis(1-methylethenyl)-ciclobutane. 
B2 (Fig. 6) is characterised by a high amount of octyl acetate. This compound was identified in each analysed beer, but at lower levels. Also 3-methyl-1-butanol, isoamyl acetate and ethyl octanoate, are present in significantly higher amounts, when compared to B1, B3, B4 and B5 beer samples. $p$-Xylene, butanal, 2-ethyl-hexanal, 3-octanone, $\beta$-cymene, 2-methyl furan and 3-methyl-2(5H)-furanone, are only present in B2 beer type.
In $\mathrm{B} 3$ beer, 35 volatile and semi-volatile organic compounds were identified. As Fig. 6 shows, the most intense peaks correspond to ethyl octanoate, isoamyl acetate, ethyl decanoate, 3-methyl-1-butanol, ethyl hexanoate and 2-phenylethyl acetate. Isopentyl hexanoate can be used to differentiate this beer type from B1, B2, B4 and B5 samples, since it was the only beer where this compound was detected at high levels. Isoamyl acetate, 3-methyl-1-butanol, $\beta$-phenylethanol,

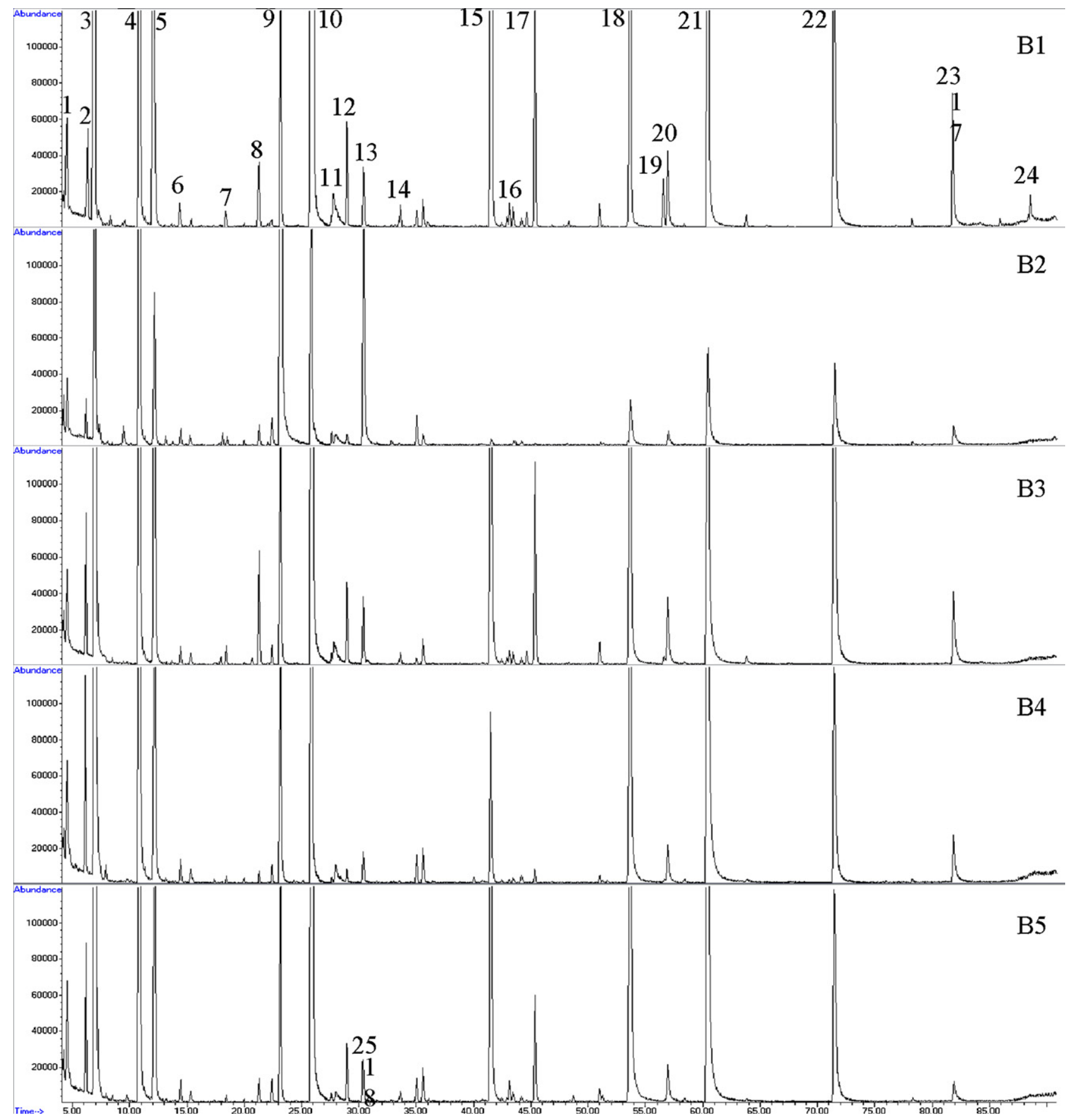

Fig. 6 - GC-qMSD profile of the different beers after headspace SPME using DVB/CAR/PDMS fibre (extraction temperature: $30{ }^{\circ} \mathrm{C}$; extraction time: $60 \mathrm{~min} ; 30 \%$ (w/v) NaCl; stirring: $\left.750 \mathrm{rpm}\right)$. Peak identification: (1) ethyl butanoate; (2)

2-methyl-1-propanol; (3) isoamyl acetate; (4) 3-methyl-1-butanol; (5) ethyl hexanoate; (6) hexyl acetate; (7) 3-octanol (IS); (8) ethyl octanoate; (9) octyl acetate; (10) 2-ethyl-1-hexanol; (11) acetic acid; (12) octyl acetate; (13) 2-ethyl-1-hexanol; (14) ethyl nonanoate; (15) ethyl decanoate; (16) decyl acetate; (17) ethyl 9-decenoate; (18) 2-phenylethyl acetate; (19) ethyl dodecanoate; (20) hexanoic acid; (21) $\beta$-phenylethanol; (22) octanoic acid; (23) n-decanoic acid; (24) 5-hydroxymethyl furfural; (25) decanal. 


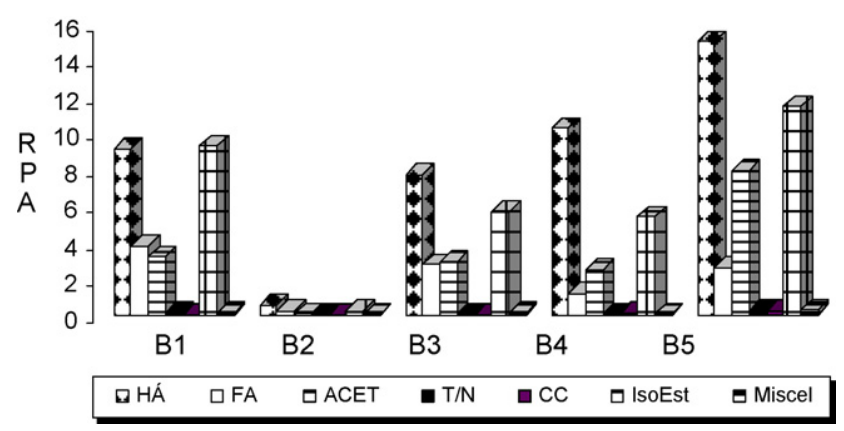

Fig. 7 - Profiles of the major volatiles found in B1-B5 beer samples (HA: higher alcohols; FA: fatty acids; ACET: higher alcohol acetates; $\mathrm{T} / \mathrm{N}$ : terpenopid and $\mathrm{C}_{13}$-norisoprenoids; CC: carbonyl compounds; IsoEst: isoamyl esters; Miscel: miscellaneous).

ethyl octanoate and 2-phenylethyl acetate, are the dominating components found in the B4 sample. Thirty-two compounds were positively identified in this sample with the experimental conditions used. Ethyl 3-hexenoate, isomenthone and (2R-cis)-5-methyl-2-(1-methylethyl)cyclohexanone, has been only detected in this beer sample. Ethyl octanoate and isoamyl acetate are the main volatiles found in "B5" beer. Also, 3-methyl-1-butanol, ethyl decanoate and ethyl hexanoate, are present in high amounts. 3-Methyl-1-hexanol, 3-methylhepthyl acetate, cadinene and 1,7-heptanediol, were only found in this beer sort. This sample is the richest in volatile and semi-volatile content, as 40 compounds were identified.

\subsubsection{Whisky volatile profile}

A typical total ion chromatogram (HS-SPME CAR/PDMS $^{-}$ GC-qMSD) obtained for the studied whisky samples, is shown in Fig. 9. More than 100 compounds were identified in all whisky samples (Table 8), from several chemical families. The differences observed according to the whisky sample were mainly quantitative. The abundance of the different volatile components (i) extracted by HS-SPME were calculated as relative peak areas $\left(R P A_{i}\right)$, defined as the ratio between the component peak area $\left(A_{i}\right)$ and the peak area $\left(A_{t}\right)$ : RPA $(\%)=A_{i} / A_{t}$. The relative standard deviation (R.S.D.\%), for the different RPA values, ranged from 10.9\% (SW1) to 12.8\% (SW2), respectively. The total free fraction, in terms of RPA, of SW4 whisky (8275.0) was 5.8 times higher than SW2 (1422.3), 3.4 than SW1 (2451.6) and 2.5 times than SW3 (3332.4). Typical profiles of studied whisky samples are shown in Fig. 9.

Quantitatively, the ethyl esters are the largest group of the studied whisky volatile constituents: $93.1 \%$ in SW1, 90.3 in SW2, $91.3 \%$ in SW3, 94.5\% in SW4 and $88.6 \%$ in SW5. These compounds are produced from ethanolysis of acylCoA, which is formed during fatty acids synthesis or degradation. These compounds make a positive contribution to the general quality of whisky, being responsible for their "fruity" and "floral" sensory properties. The ethyl esters from C8, C10 and C12 fatty acids, which contribute with sweet and fruity notes, and isoamyl alcohols, represent the major compounds in either of the analysed whiskys (Fig. 10). The relative amount of ethyl esters in the different types of studied whiskys was reasonably constant and the differences were not significant. As for ethyl esters of diprotic acids it is noticed that the relative amount of diethyl succinate is much higher than that found for ethyl lactate. Similar contents of diethyl succinate were observed in all studied whisky samples.

The higher alcohols fraction is composed mainly by $n$ alcohols of $\mathrm{C}_{6}$ chain length and aromatic compounds such as $\beta$-phenylethanol. The presence of these compounds may cause a "flowery" and "sweet" note, which could be considered as a positive characteristic for whisky. The alcohol fraction of SW3 and SW4 (RPA=192.2 and 143.8, respectively) was significantly different at the $95 \%$ level from the other studied whiskys, which present RPA values of 50.7, 48.3 and 24.9 for SW1, SW2 and SW5, respectively. 3-Methylbutan-1-ol and 2methyl-1-propanol were markedly the most abundant higher alcohols. The content of isoamyl alcohol was notably higher in the SW1 whisky contrarily to SW3 that presents the lowest content.

The third class of compounds, in terms of quantitative volatile composition, are fatty acids The most important fatty acids present in the whisky samples studied were C8, $\mathrm{C} 10$ and C12. Fatty acids content in the analysed whisky samples was not significantly different at the 95\% level: $1.4 \%$ in SW1, 1.7\% in SW2, 1.3 in SW3, 2.1\% in SW4 and $1.6 \%$ in SW5 samples. The carbonyl compounds include
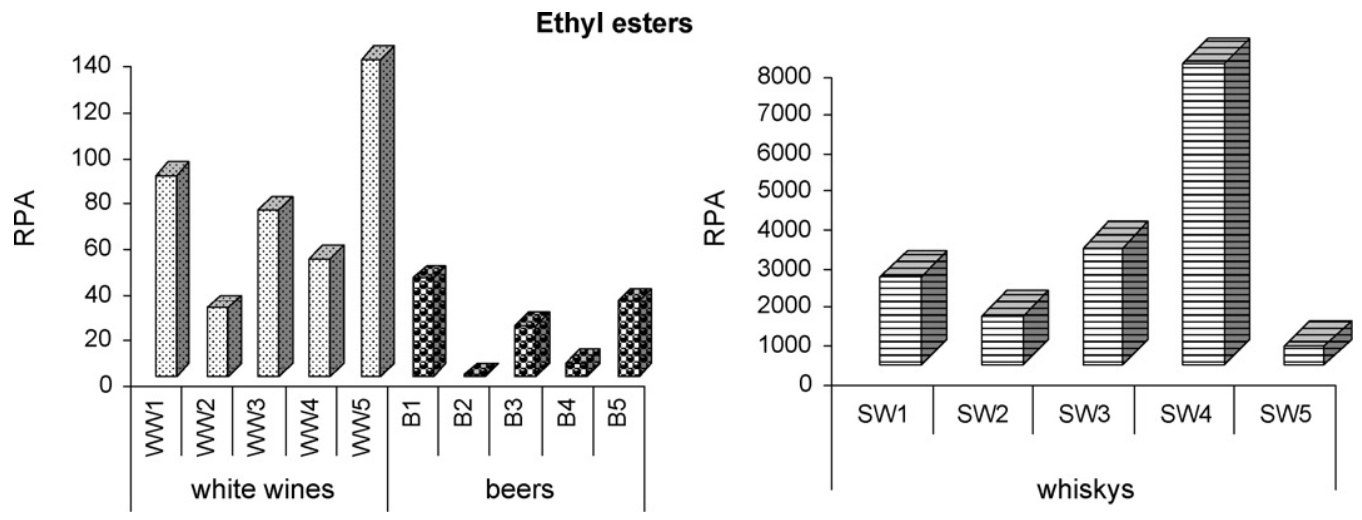

Fig. 8 - Amount of ethyl esters found in studied alcoholic beverages. 
Table 8 - VOCs and SVOCs identified and quantified (RPA, \%) in different whisky samples after dynamic HS-SPME extraction using CAR/PDMS coating and GC-qMSD analysis (extraction temperature: $40^{\circ} \mathrm{C}$; extraction time: $60 \mathrm{~min}$, sample with $30 \%, \mathrm{w} / \mathrm{v}$, NaCl)

\begin{tabular}{|c|c|c|c|c|c|c|c|c|c|c|c|c|}
\hline \multirow[t]{2}{*}{$\mathrm{RT}$ (min) } & \multirow[t]{2}{*}{$\mathrm{KI}^{\mathrm{a}}$} & \multirow[t]{2}{*}{ Compound } & \multicolumn{2}{|c|}{ SW1 } & \multicolumn{2}{|c|}{ SW2 } & \multicolumn{2}{|c|}{ SW3 } & \multicolumn{2}{|c|}{ SW4 } & \multicolumn{2}{|c|}{ SW5 } \\
\hline & & & $\begin{array}{c}\text { Average } \\
\text { peak area }\end{array}$ & $\operatorname{RPA}^{\mathrm{c}}(\%)$ & $\begin{array}{c}\text { Average } \\
\text { Peak area }\end{array}$ & RPA (\%) & $\begin{array}{c}\text { Average } \\
\text { peak area }\end{array}$ & RPA (\%) & $\begin{array}{c}\text { Average } \\
\text { peak area }\end{array}$ & RPA (\%) & $\begin{array}{c}\text { Average } \\
\text { peak area }\end{array}$ & RPA (\%) \\
\hline 4.551 & 1079 & Ethyl butyrate & $7.47 \times 10^{5}$ & 0.02 & $1.54 \times 10^{6}$ & 0.04 & $-d$ & - & - & - & - & - \\
\hline 4.753 & 1089 & 1-Propanol & $1.14 \times 10^{7}$ & 0.30 & $2.56 \times 10^{6}$ & 0.04 & $3.66 \times 10^{6}$ & 0.22 & $7.96 \times 10^{6}$ & 0.46 & $1.02 \times 10^{7}$ & 0.43 \\
\hline 4.933 & 1097 & Ethyl 2-methylbutyrate & $1.39 \times 10^{6}$ & 0.03 & $4.22 \times 10^{5}$ & 0.01 & - & - & - & - & - & - \\
\hline 5.309 & 1112 & Ethyl 3-methylbutanoate & $2.44 \times 10^{6}$ & 0.05 & - & - & - & - & - & - & - & - \\
\hline 5.349 & 1114 & Ethyl isovalerate & - & - & $1.48 \times 10^{6}$ & 0.04 & - & - & - & - & - & - \\
\hline 5.633 & 1125 & Hexanal & - & - & - & - & - & - & - & - & $1.05 \times 10^{6}$ & 0.04 \\
\hline 6.470 & 1153 & 2-Methyl-1-propanol & $3.84 \times 10^{7}$ & 0.95 & $9.31 \times 10^{6}$ & 0.17 & $6.42 \times 10^{6}$ & 0.38 & $1.00 \times 10^{7}$ & 0.57 & $1.65 \times 10^{7}$ & 0.70 \\
\hline 6.708 & 1161 & Ethylbenzene & - & - & - & - & - & - & - & - & $2.24 \times 10^{5}$ & 0.01 \\
\hline 6.829 & 1165 & Isoamyl acetate & $8.82 \times 10^{6}$ & 0.17 & $1.25 \times 10^{7}$ & 0.32 & $9.13 \times 10^{6}$ & 0.54 & $2.46 \times 10^{7}$ & 1.40 & $1.19 \times 10^{7}$ & 0.50 \\
\hline 7.130 & 1173 & -Xylene & $3.63 \times 10^{5}$ & 0.01 & - & - & - & - & - & - & $3.08 \times 10^{6}$ & 0.13 \\
\hline 7.904 & 1195 & 1-Butanol & $6.83 \times 10^{5}$ & 0.01 & $2.75 \times 10^{5}$ & 0.00 & - & - & - & - & - & - \\
\hline 9.013 & 1225 & o-Xylene & - & - & - & - & - & - & - & - & $1.68 \times 10^{6}$ & 0.07 \\
\hline 9.267 & 1231 & 5-Methyl-2-hexanone & $5.82 \times 10^{5}$ & 0.01 & - & - & - & - & - & - & - & - \\
\hline 9.653 & 1240 & Limonene & - & - & $1.96 \times 10^{5}$ & 0.00 & - & - & - & - & - & - \\
\hline 11.186 & 1275 & 3-Methyl-1-butanol & $3.06 \times 10^{8}$ & 7.15 & $7.36 \times 10^{7}$ & 1.33 & $2.28 \times 10^{7}$ & 1.34 & $3.20 \times 10^{7}$ & 1.83 & $7.51 \times 10^{7}$ & 3.19 \\
\hline 11.948 & 1290 & Ethyl hexanoate & $5.18 \times 10^{7}$ & 1.21 & $3.91 \times 10^{7}$ & 0.98 & $9.93 \times 10^{6}$ & 0.59 & $1.32 \times 10^{7}$ & 0.75 & $2.69 \times 10^{7}$ & 1.13 \\
\hline 12.938 & 1310 & Styrene & $2.37 \times 10^{6}$ & 0.05 & $2.21 \times 10^{6}$ & 0.05 & $6.84 \times 10^{6}$ & 0.40 & $7.19 \times 10^{6}$ & 0.41 & $2.69 \times 10^{7}$ & 2.09 \\
\hline 13.314 & 1318 & 1-Pentanol & $9.57 \times 10^{5}$ & 0.02 & - & - & - & - & - & - & - & - \\
\hline 14.033 & 1333 & 1,2,4-Trimethylbenzene & $7.45 \times 10^{5}$ & 0.02 & - & - & $1.80 \times 10^{5}$ & 0.01 & $3.77 \times 10^{5}$ & 0.03 & $1.17 \times 10^{6}$ & 0.05 \\
\hline 14.242 & 1337 & Hexyl acetate & $4.78 \times 10^{5}$ & 0.01 & $1.20 \times 10^{6}$ & 0.03 & $2.52 \times 10^{5}$ & 0.01 & $4.91 \times 10^{5}$ & 0.02 & - & - \\
\hline 15.442 & 1360 & 3-Cyclohexene-1-carboxylic acid & $5.72 \times 10^{5}$ & 0.01 & $3.24 \times 10^{5}$ & 0.01 & $1.75 \times 10^{5}$ & 0.01 & $9.61 \times 10^{6}$ & 0.05 & $7.51 \times 10^{5}$ & 0.03 \\
\hline 16.655 & 1381 & 1,1-Diethoxypropane & $1.17 \times 10^{5}$ & 0.00 & - & - & - & 0.05 & - & - & - & - \\
\hline 17.478 & 1395 & 1-Ethyl-3-methylbenzene & $6.76 \times 10^{5}$ & 0.02 & $5.39 \times 10^{5}$ & 0.01 & - & - & - & - & $9.46 \times 10^{5}$ & 0.04 \\
\hline 18.227 & 1409 & Ethyl heptanoate & $2.52 \times 10^{6}$ & 0.06 & $1.92 \times 10^{6}$ & 0.05 & - & - & $2.64 \times 10^{5}$ & 0.02 & $1.28 \times 10^{6}$ & 0.05 \\
\hline 18.426 & 1413 & Ethyl pentanoate & - & - & - & - & $2.57 \times 10^{5}$ & 0.02 & - & - & - & - \\
\hline 18.753 & 1416 & 1-Methyl-3-(1-methylethyl)benzene & $1.60 \times 10^{5}$ & 0.00 & - & - & - & - & - & - & - & - \\
\hline 19.276 & 1429 & 2,3-Butanediol & $6.55 \times 10^{5}$ & 0.02 & - & - & - & - & - & - & - & - \\
\hline 19.547 & 1434 & Hexyl hexanoate & $1.19 \times 10^{5}$ & 0.00 & - & - & - & - & - & - & - & - \\
\hline 20.100 & 1445 & 1-Hexanol & $2.30 \times 10^{6}$ & 0.05 & $6.09 \times 10^{5}$ & 0.01 & - & - & - & - & $7.24 \times 10^{5}$ & 0.03 \\
\hline 21.299 & 1466 & Heptyl acetate & - & - & $2.07 \times 10^{5}$ & 0.00 & - & - & - & - & $2.14 \times 10^{5}$ & 0.01 \\
\hline 21.943 & 1477 & 2-Nonanone & $6.30 \times 10^{5}$ & 0.01 & - & - & - & - & - & - & - & - \\
\hline 22.071 & 1479 & 5-Methyl-2-hexanone & - & - & $4.22 \times 10^{5}$ & 0.01 & - & - & - & - & $7.47 \times 10^{5}$ & 0.02 \\
\hline 22.250 & 1482 & (E)-2-Nonen-1-ol & $3.72 \times 10^{5}$ & 0.01 & - & - & - & - & - & - & $1.09 \times 10^{6}$ & 0.04 \\
\hline 23.617 & 1505 & 2,4,6-Trimethyloctane & - & - & - & - & - & - & - & - & $5.58 \times 10^{6}$ & 0.02 \\
\hline 23.794 & 1509 & Tetradecane & - & - & - & - & - & - & - & - & $7.99 \times 10^{6}$ & 0.03 \\
\hline 25.892 & 1547 & Ethyl octanoate & $9.72 \times 10^{8}$ & 22.56 & $8.16 \times 10^{8}$ & 20.08 & $2.44 \times 10^{8}$ & 15.10 & $2.54 \times 10^{8}$ & 14.44 & $9.19 \times 10^{8}$ & 38.50 \\
\hline 27.513 & 1575 & Isopentyl hexanoate & $1.05 \times 10^{6}$ & 0.02 & $7.54 \times 10^{5}$ & 0.02 & $3.02 \times 10^{5}$ & 0.02 & $4.86 \times 10^{5}$ & 0.03 & $1.05 \times 10^{6}$ & 0.04 \\
\hline 27.967 & 1582 & Furfural & $1.56 \times 10^{7}$ & 0.36 & $1.11 \times 10^{7}$ & 0.29 & $3.10 \times 10^{6}$ & 0.18 & $4.89 \times 10^{6}$ & 0.28 & $7.13 \times 10^{6}$ & 0.30 \\
\hline 28.275 & 1587 & 1-Ethyl-2,3-dimethylbenzene & $6.45 \times 10^{5}$ & 0.01 & - & - & $1.94 \times 10^{5}$ & 0.01 & - & - & - & - \\
\hline 28.405 & 1589 & 1,2,3,4-Tetramethylbenzene & - & - & $7.34 \times 10^{5}$ & 0.02 & - & - & - & - & - & - \\
\hline 28.830 & 1596 & $n$-Octyl acetate & $3.38 \times 10^{5}$ & 0.01 & $5.64 \times 10^{5}$ & 0.01 & $2.68 \times 10^{5}$ & 0.02 & $6.65 \times 10^{5}$ & 0.04 & $1.25 \times 10^{6}$ & 0.05 \\
\hline
\end{tabular}


Table 8 (Continued)

\begin{tabular}{|c|c|c|c|c|c|c|c|c|c|c|c|c|}
\hline \multirow[t]{2}{*}{$\mathrm{RT}(\min )$} & \multirow[t]{2}{*}{$\mathrm{KI}^{\mathrm{a}}$} & \multirow[t]{2}{*}{ Compound } & \multicolumn{2}{|c|}{ SW1 } & \multicolumn{2}{|c|}{ SW2 } & \multicolumn{2}{|c|}{ SW3 } & \multicolumn{2}{|c|}{ SW4 } & \multicolumn{2}{|c|}{ SW5 } \\
\hline & & & $\begin{array}{c}\text { Average } \\
\text { peak area }\end{array}$ & $\mathrm{RPA}^{\mathrm{c}}(\%)$ & $\begin{array}{c}\text { Average } \\
\text { Peak area }\end{array}$ & RPA (\%) & $\begin{array}{c}\text { Average } \\
\text { peak area }\end{array}$ & RPA (\%) & $\begin{array}{c}\text { Average } \\
\text { peak area }\end{array}$ & RPA (\%) & $\begin{array}{c}\text { Average } \\
\text { peak area }\end{array}$ & RPA (\%) \\
\hline 29.611 & 1608 & Ethyl pentanoate & $2.03 \times 10^{5}$ & 0.00 & - & - & - & - & - & - & - & - \\
\hline 29.693 & 1609 & Ethyl 6-heptenoate & - & - & $1.63 \times 10^{5}$ & 0.00 & - & - & - & - & - & - \\
\hline 31.419 & 1635 & Benzaldehyde & $4.91 \times 10^{6}$ & 0.11 & $3.41 \times 10^{6}$ & 0.09 & $9.07 \times 10^{5}$ & 0.05 & $1.49 \times 10^{6}$ & 0.09 & $4.45 \times 10^{6}$ & 0.18 \\
\hline 32.203 & 1646 & Hexyl octanoate & $6.06 \times 10^{5}$ & 0.02 & - & - & - & - & $1.97 \times 10^{6}$ & 0.11 & - & - \\
\hline 32.269 & 1647 & Propyl octanoate & - & - & $6.04 \times 10^{5}$ & 0.01 & - & - & - & - & $7.78 \times 10^{5}$ & 0.03 \\
\hline 32.776 & 1654 & Heptanal & - & - & - & - & - & - & $2.32 \times 10^{5}$ & 0.01 & - & - \\
\hline 33.593 & 1665 & Ethyl nonanoate & $8.65 \times 10^{6}$ & 0.20 & $7.20 \times 10^{6}$ & 0.17 & $2.99 \times 10^{6}$ & 0.18 & $2.46 \times 10^{6}$ & 0.14 & $7.89 \times 10^{6}$ & 0.33 \\
\hline 34.930 & 1683 & Isobutyl octanoate & $2.02 \times 10^{6}$ & 0.05 & $1.95 \times 10^{6}$ & 0.05 & $7.34 \times 10^{5}$ & 0.03 & $9.00 \times 10^{5}$ & 0.05 & $1.80 \times 10^{6}$ & 0.08 \\
\hline 35.518 & 1690 & 1-Octanol & $8.39 \times 10^{5}$ & 0.02 & - & - & - & - & $3.14 \times 10^{6}$ & 0.21 & $1.36 \times 10^{6}$ & 0.06 \\
\hline 35.551 & 1691 & 1-Hexene & - & - & $3.14 \times 10^{5}$ & 0.01 & $1.07 \times 10^{5}$ & 0.01 & - & - & - & - \\
\hline 35.574 & 1691 & 1,2-Diethyl-cyclobutane & - & - & - & - & - & - & $2.18 \times 10^{5}$ & 0.01 & - & - \\
\hline 38.028 & 1868 & Methyl butanoate & - & - & - & - & $2.31 \times 10^{5}$ & 0.01 & - & - & - & - \\
\hline 38.051 & 1869 & 2-Dodecanone & $9.36 \times 10^{5}$ & 0.02 & - & - & - & - & $5.01 \times 10^{6}$ & 0.33 & $7.47 \times 10^{5}$ & 0.03 \\
\hline 38.064 & 1869 & Methyl nonoate & - & - & $4.73 \times 10^{5}$ & 0.01 & - & - & - & - & - & - \\
\hline 39.29 & 1891 & Hexyl hexanoate & - & - & - & - & - & - & $4.77 \times 10^{5}$ & 0.02 & - & - \\
\hline 39.944 & 1903 & Ethyl-2-furoate & $2.88 \times 10^{5}$ & 0.01 & $1.47 \times 10^{5}$ & 0.00 & $9.55 \times 10^{4}$ & 0.01 & - & - & $3.73 \times 10^{5}$ & 0.02 \\
\hline 41.764 & 1940 & Ethyl decanoate & $2.18 \times 10^{9}$ & 50.81 & $2.18 \times 10^{9}$ & 55.42 & $1.06 \times 10^{9}$ & 62.58 & $1.02 \times 10^{9}$ & 58.00 & $1.11 \times 10^{9}$ & 47.08 \\
\hline 42.510 & 1955 & Ethyl benzoate & $1.05 \times 10^{7}$ & 0.25 & - & - & $1.06 \times 10^{6}$ & 0.06 & $1.39 \times 10^{6}$ & 0.08 & $3.60 \times 10^{6}$ & 0.15 \\
\hline 43.013 & 1965 & Isoamyl octanoate & - & - & $1.21 \times 10^{7}$ & 0.30 & $7.83 \times 10^{6}$ & 0.46 & $8.45 \times 10^{6}$ & 0.48 & $3.96 \times 10^{6}$ & 0.17 \\
\hline 43.418 & 1972 & Decanyl acetate & - & - & - & - & $6.82 \times 10^{5}$ & 0.04 & - & - & - & - \\
\hline 44.513 & 1993 & Diethyl succinate & $6.09 \times 10^{5}$ & 0.02 & $2.49 \times 10^{5}$ & 0.00 & - & - & - & - & - & - \\
\hline 44.706 & 1997 & Decyl acetate & $7.97 \times 10^{5}$ & 0.02 & $9.16 \times 10^{5}$ & 0.02 & $7.18 \times 10^{5}$ & 0.04 & $1.36 \times 10^{6}$ & 0.08 & $5.86 \times 10^{5}$ & 0.02 \\
\hline 44.945 & 2001 & 2,5-Diethyl heptanoic acid & - & - & $3.06 \times 10^{5}$ & 0.01 & $1.40 \times 10^{5}$ & 0.01 & - & - & - & - \\
\hline 45.392 & 2009 & Ethyl 9-decenoate & $2.50 \times 10^{7}$ & 0.58 & $4.67 \times 10^{7}$ & 1.18 & $8.61 \times 10^{6}$ & 0.51 & $3.43 \times 10^{6}$ & 0.17 & $1.03 \times 10^{7}$ & 0.43 \\
\hline 46.831 & 2039 & Naphthalene & $3.69 \times 10^{6}$ & 0.09 & $2.77 \times 10^{6}$ & 0.08 & $1.38 \times 10^{6}$ & 0.08 & $4.89 \times 10^{5}$ & 0.03 & - & - \\
\hline 47.403 & 2051 & $\mathrm{TDN}^{\mathrm{e}}$ & $3.31 \times 10^{5}$ & 0.01 & $3.14 \times 10^{5}$ & 0.01 & - & - & - & - & - & - \\
\hline 47.873 & 2062 & Ethyl propionate & $1.32 \times 10^{6}$ & 0.03 & $1.34 \times 10^{6}$ & 0.03 & $5.45 \times 10^{5}$ & 0.03 & $8.30 \times 10^{5}$ & 0.05 & - & - \\
\hline 49.164 & 2085 & Ethyl undecanoate & $1.78 \times 10^{6}$ & 0.04 & $1.46 \times 10^{6}$ & 0.03 & $5.73 \times 10^{5}$ & 0.03 & $6.16 \times 10^{5}$ & 0.03 & - & - \\
\hline 50.250 & 2106 & Butyl caprate & $3.10 \times 10^{6}$ & 0.07 & $3.51 \times 10^{6}$ & 0.09 & $1.63 \times 10^{6}$ & 0.08 & $2.01 \times 10^{6}$ & 0.11 & - & - \\
\hline 50.975 & 2122 & 1-Decanol & $2.49 \times 10^{6}$ & 0.05 & - & - & $9.46 \times 10^{5}$ & 0.04 & - & - & - & - \\
\hline 51.024 & 2124 & 1-Ethyl-2-heptyl-cyclopropane & - & - & $1.35 \times 10^{6}$ & 0.03 & - & - & $1.52 \times 10^{6}$ & 0.10 & - & - \\
\hline 51.704 & 2139 & Ethyl phenyl acetate & - & - & $2.28 \times 10^{5}$ & 0.01 & - & - & - & - & $1.82 \times 10^{7}$ & 0.77 \\
\hline 53.642 & 2181 & 2-Phenylethyl acetate & $5.77 \times 10^{6}$ & 0.14 & $1.19 \times 10^{7}$ & 0.31 & $9.92 \times 10^{6}$ & 0.59 & $1.95 \times 10^{7}$ & 0.99 & - & - \\
\hline 54.698 & 2204 & 1-Methyl naphthalene & $6.10 \times 10^{5}$ & 0.01 & $5.47 \times 10^{5}$ & 0.01 & - & - & - & - & - & - \\
\hline 56.682 & 2248 & Ethyl dodecanoate & $5.37 \times 10^{8}$ & 12.59 & $5.76 \times 10^{8}$ & 15.72 & $2.37 \times 10^{8}$ & 13.99 & $2.86 \times 10^{8}$ & 16.16 & $1.57 \times 10^{7}$ & 0.67 \\
\hline 57.862 & 2274 & Isoamyl decanoate & $7.97 \times 10^{6}$ & 0.17 & $1.01 \times 10^{7}$ & 0.27 & $5.01 \times 10^{6}$ & 0.30 & $7.64 \times 10^{6}$ & 0.49 & - & - \\
\hline 58.402 & 2285 & Ethyl hydrocinnamate & $2.70 \times 10^{5}$ & 0.01 & - & - & $1.67 \times 10^{5}$ & 0.01 & - & - & - & - \\
\hline 59.559 & 2309 & Tetrahydro-3-furanmethanol & - & - & - & - & $2.87 \times 10^{5}$ & 0.02 & - & - & - & - \\
\hline 59.644 & 2311 & 1-Tridecene & - & - & $1.03 \times 10^{6}$ & 0.02 & - & - & - & - & - & - \\
\hline 60.170 & 2322 & Ethyl undecylenate & - & - & $1.17 \times 10^{6}$ & 0.03 & - & - & - & - & - & - \\
\hline 60.441 & 2328 & $\beta$-Phenylethanol & $4.08 \times 10^{6}$ & 0.08 & $3.15 \times 10^{6}$ & 0.10 & $1.37 \times 10^{6}$ & 0.08 & $1.97 \times 10^{6}$ & 0.11 & $3.70 \times 10^{6}$ & 0.16 \\
\hline 65.397 & 2427 & Octyl cyclopropane & $1.20 \times 10^{6}$ & 0.02 & $1.02 \times 10^{6}$ & 0.03 & $1.03 \times 10^{6}$ & 0.06 & - & - & $9.48 \times 10^{5}$ & 0.04 \\
\hline
\end{tabular}


Table 8 (Continued)

\begin{tabular}{|c|c|c|c|c|c|c|c|c|c|c|c|c|c|}
\hline \multirow[t]{2}{*}{$\mathrm{RT}(\min )$} & \multirow[t]{2}{*}{$\mathrm{KI}^{\mathrm{a}}$} & \multirow[t]{2}{*}{ Compound } & \multirow[t]{2}{*}{ Identity $^{b}$} & \multicolumn{2}{|c|}{ SW1 } & \multicolumn{2}{|c|}{ SW2 } & \multicolumn{2}{|c|}{ SW3 } & \multicolumn{2}{|c|}{ SW4 } & \multicolumn{2}{|c|}{ SW5 } \\
\hline & & & & $\begin{array}{c}\text { Average } \\
\text { peak area }\end{array}$ & $\operatorname{RPA}^{\mathrm{C}}(\%)$ & $\begin{array}{c}\text { Average } \\
\text { Peak area }\end{array}$ & RPA (\%) & $\begin{array}{c}\text { Average } \\
\text { peak area }\end{array}$ & RPA (\%) & $\begin{array}{c}\text { Average } \\
\text { peak area }\end{array}$ & RPA (\%) & $\begin{array}{c}\text { Average } \\
\text { peak area }\end{array}$ & RPA (\%) \\
\hline 65.446 & 2428 & Cyclodecane & & - & - & - & - & - & - & $1.64 \times 10^{6}$ & 0.08 & - & - \\
\hline 67.315 & 2466 & Phenol & & - & - & - & - & - & - & - & - & $2.49 \times 10^{6}$ & 0.05 \\
\hline 70.267 & 2512 & $\beta$-Farnesene & & - & - & $4.19 \times 10^{5}$ & 0.01 & $3.82 \times 10^{5}$ & 0.02 & $5.71 \times 10^{5}$ & 0.03 & - & - \\
\hline 70.630 & 2516 & Ethyl tetradecanoate & & $3.85 \times 10^{6}$ & 0.06 & $6.11 \times 10^{6}$ & 0.18 & $3.14 \times 10^{6}$ & 0.19 & $3.14 \times 10^{6}$ & 0.21 & - & - \\
\hline 71.496 & 2524 & Octanoic acid & & $1.95 \times 10^{7}$ & 0.36 & $1.51 \times 10^{7}$ & 0.45 & $4.98 \times 10^{6}$ & 0.29 & $1.97 \times 10^{6}$ & 0.21 & $9.39 \times 10^{6}$ & 0.40 \\
\hline 72.780 & 2537 & (E)-11-Tetradecanoic acid & & - & - & $1.42 \times 10^{6}$ & 0.04 & - & - & - & - & - & - \\
\hline 77.915 & 2679 & Cyclododecane & & - & - & - & - & $3.00 \times 10^{6}$ & 0.18 & - & - & - & - \\
\hline 77.932 & 2679 & 1-Hexadecanol & & - & - & $2.31 \times 10^{6}$ & 0.08 & - & - & - & - & - & - \\
\hline 77.971 & 2680 & (E)-5-Octadecene & & $2.54 \times 10^{6}$ & 0.04 & - & - & - & - & $5.01 \times 10^{6}$ & 0.33 & - & - \\
\hline 79.449 & 2709 & 3-(1-Methylethyl)phenol & & - & - & - & - & - & - & - & - & $2.38 \times 10^{5}$ & 0.00 \\
\hline 81.161 & 2747 & Ethyl octadecanoate & & - & - & - & - & $6.81 \times 10^{5}$ & 0.04 & - & - & - & - \\
\hline 81.197 & 2748 & Ethyl isohexanoate & & $7.96 \times 10^{5}$ & 0.02 & - & - & - & - & $1.14 \times 10^{6}$ & 0.06 & - & - \\
\hline 81.959 & 2765 & Decanoic acid & & $4.67 \times 10^{7}$ & 0.85 & $4.68 \times 10^{7}$ & 1.44 & $1.42 \times 10^{7}$ & 0.84 & $1.66 \times 10^{7}$ & 0.95 & $2.34 \times 10^{7}$ & 1.00 \\
\hline 83.391 & 2795 & 2,4-Bis-(1,1dimethylethyl)phenol & & - & - & - & - & - & - & - & - & $2.34 \times 10^{7}$ & 0.02 \\
\hline 87.195 & 2866 & Benzenecarboxylic acid & & - & - & - & - & - & - & - & - & $7.60 \times 10^{5}$ & 0.01 \\
\hline 87.682 & 2876 & 1,2,4,5-Tetramethylbenzene & & - & - & - & - & - & - & - & - & $6.81 \times 10^{5}$ & 0.01 \\
\hline 88.467 & 2892 & Dodecanoic acid & & $5.25 \times 10^{6}$ & 0.07 & $5.45 \times 10^{6}$ & 0.17 & $4.24 \times 10^{6}$ & 0.25 & $1.97 \times 10^{6}$ & 0.52 & $3.02 \times 10^{6}$ & 0.13 \\
\hline 89.251 & 2907 & 5-(Hydroxymethyl)furfural & & - & - & - & - & - & - & - & - & $3.02 \times 10^{6}$ & 0.13 \\
\hline 90.235 & 2926 & 4-Hydroxy-benzene ethanol & & - & - & - & - & - & - & - & - & $7.42 \times 10^{6}$ & 0.14 \\
\hline \multicolumn{4}{|c|}{ Total compounds identified per whisky } & \multicolumn{2}{|l|}{32} & \multicolumn{2}{|l|}{36} & \multicolumn{2}{|l|}{32} & \multicolumn{2}{|l|}{31} & \multicolumn{2}{|l|}{26} \\
\hline \multicolumn{4}{|c|}{ Sum } & \multicolumn{2}{|l|}{$4.31 \times 10^{9}$} & \multicolumn{2}{|l|}{$2.95 \times 10^{9}$} & \multicolumn{2}{|l|}{$1.68 \times 10^{9}$} & \multicolumn{2}{|l|}{$1.79 \times 10^{9}$} & \multicolumn{2}{|l|}{$2.38 \times 10^{9}$} \\
\hline \multicolumn{4}{|c|}{ R.S.D. $(\%)$ on $\operatorname{sum}(n=4)$} & \multicolumn{2}{|l|}{10.9} & \multicolumn{2}{|l|}{12.8} & \multicolumn{2}{|l|}{11.4} & \multicolumn{2}{|l|}{11.2} & \multicolumn{2}{|l|}{12.6} \\
\hline
\end{tabular}

${ }^{a}$ Experimentally determined Kovàts indices on the BP20 column, relative to $\mathrm{C}_{8}-\mathrm{C}_{20}$ hydrocarbons.

b A, components identified on the basis of the retention time and EI mass spectra of pure standard; B, components identified on the basis of their EI mass spectra only. c Percent relative area.

d Not detected.

e 1,1,6-Trimethyl-1,2-dihydro-naphtalene. 


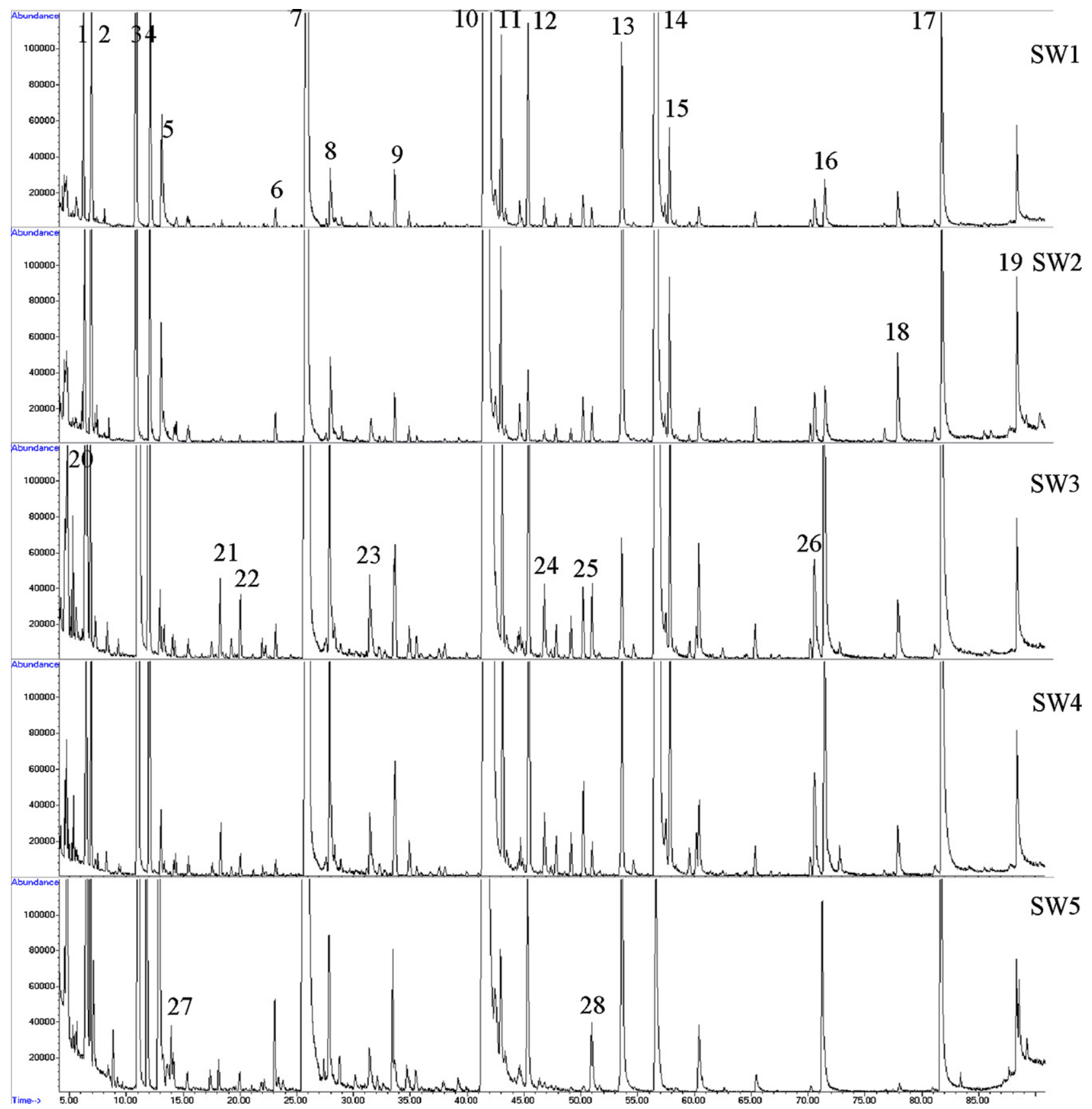

Fig. 9 - Comparison of GC-qMS chromatograms of commercial whisky samples obtained by headspace SPME using CAR/PDMS fibre (extraction temperature: $40^{\circ} \mathrm{C}$; extraction time: $60 \mathrm{~min}$; $30 \%$ (w/v) NaCl; stirring: $750 \mathrm{rpm}$ ). Peak identification: (1) 2-methyl-1-propanol; (2) isoamyl acetate; (3) 3-methyl-1-butanol; (4) ethyl hexanoate; (5) styrene; (6) 3-octanol (IS); (7) ethyl octanoate; (8) furfural; (9) ethyl nonanoate; (10) ethyl decanoate; (11) isoamyl octanoate; (12) ethyl 9-decenoate; (13) 2-phenylethyl acetate; (14) ethyl dodecanoate; (15) isoamyl decanoate; (16) octanoic acid; (17) n-decanoic acid; (18) cyclododecane; (19) dodecanoic acid; (20) 1-propanol; (21) ethyl heptanoate; (22) 1-hexanol; (23) benzaldehyde; (24) naphthalene; (25) isobutyl caprate; (26) ethyl tetradecanoate; (27) 1,2,3-trimethylbenzene; (28) 1-butyl-2-ethyl-cyclobutane.

aldehydes and ketones. Only few aldehydes have been detected among the whisky volatile constituents, probably because they are reduced to the corresponding alcohols. The carbonyl compounds content in SW1 (RPA=5.8), SW2 $(\mathrm{RPA}=5.4)$ SW5 $(\mathrm{RPA}=3.7)$ whisky is similar but in SW3 and SW4 samples the values are much higher 17.9 and 32.3, respectively.
Terpenoids, which may have an important contribution on the "floral" and "fruity" aromas of the whisky, and $\mathrm{C}_{13}$ norisoprenoids (derived from carotenoids degradation) that possibly contribute with "camphor", "honey-like" or "cassis" notes, are the most abundant in SW1 (RPA =10.4) and SW5 $(\mathrm{RPA}=10.9)$. Contrarily, lower levels of these compounds were found in SW3 whisky samples (RPA =1.9). 


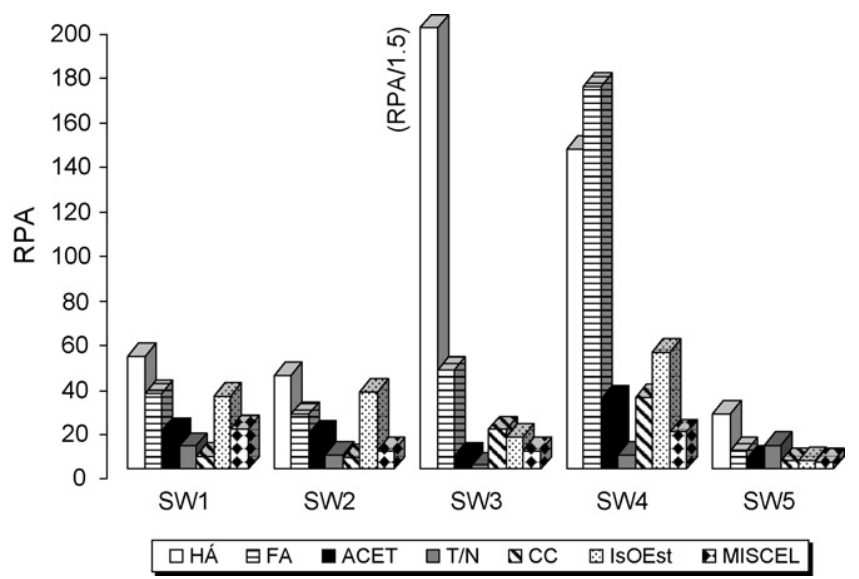

Fig. 10 - Distribution of compounds classes by whisky samples (HA: higher alcohols; FA: fatty acids; ACET: higher alcohol acetates; $\mathrm{T} / \mathrm{N}$ : terpenopid and $\mathrm{C}_{13}$-norisoprenoids; CC: carbonyl compounds; IsoEst: isoamyl esters; Miscel: miscellaneous).

\section{Conclusions}

The SPME fibre, adsorption and desorption parameters were chosen to obtain the maximum sensitivity for the greater number of compounds. The SPME fibre, adsorption and desorption parameters were chosen in order to obtain maximum sensitivity to yield a greater number of compounds. The chromatographic profiles obtained after extraction with PDMS, PA and Stableflex coatings, suggest that using a DVB/CAR/PDMS fibre provided effective sample enrichment and enabled extraction of a wide variety of compounds from beers. As for wine and whisky, the most suitable fibers for VOCs and SVOCs extraction were PDMS and CAR/PDMS, respectively. The optimal extraction conditions for selected fiber were: $30 \mathrm{ml}$ of sample, extraction time $60 \mathrm{~min}$, extraction temperature $30^{\circ} \mathrm{C}$ for wine and beer and $40^{\circ} \mathrm{C}$ for whisky, by headspace mode of a stirred sample saturated with $\mathrm{NaCl}(30 \%$, $\mathrm{w} / \mathrm{v})$.

The validated methodology provides an appropriate and selective way to characterize the volatile and semi-volatile organic compounds in wine, beer and whisky. The samples chromatographic separation enables the identification of the compounds responsible for the aroma and flavour differences in the samples.

More than 64 compounds in beer, 44 in white wines and 104 in whiskys were identified, belonging to a variety of chemical families such as, ethyl esters, higher alcohols, isoamyl acetates, fatty acids, mainly. Their relative contents were found to be different, which might lead to the differentiation of the beverages volatile profile. Qualitatively and quantitatively, ethyl esters (mainly ethyl octanoate, ethyl decanoate and ethyl dodecanoate) are the largest group of the volatile composition found in studied alcoholic beverage samples. The higher alcohols (aliphatic and aromatic) and fatty acids also constitute important groups of beverage aroma compounds.
REFERENCES

[1] A. Rapp, H. Mandery, Experientia 42 (1986) 873.

[2] S.P. Arrhenius, L. McCloskey, M. Sylvan, J. Agric. Food Chem. 44 (1996) 1085.

[3] V. Ferreira, R. Lopez, J.F. Cacho, J. Sci. Food Agric. 80 (2000) 1659.

[4] P. Romano, C. Fiore, M. Paraggio, M. Caruso, A. Capece, Int. J. Food Microbiol. 86 (2003) 169.

[5] P. Dubois, Rev. Fr. Oenol. 145 (1994) 27.

[6] F. Radler, Yeast: metabolism of organic acids, in: G.H. Fleet (Ed.), Wine Microbiology and Biotechnology, Harwood Academic Publishers, Chur, Switzerland, 1993, p. 165.

[7] K.J. Verstrepen, G. Derdelinckx, J.-P. Duffour, J. Winderickx, J.M. Velein, I.S. Pretorious, F.R. Delvaux, J. Biosci. Bioeng. 96 (2) (2003) 110.

[8] R.F. Simpson, J. Sci. Food Agric. 28 (1980) 214

[9] A. Rapp, G. Versini, In: J. Rantz (Ed.), Proceedings of the International Symposium on Nitrogen in Grapes and Wines, Seatle, WA, 1991, pp. 156-164.

[10] V. Ferreira, N. Ortin, A. Escudero, R. Lopez, J. Cacho, J. Agric. Food Chem. 50 (2002) 4048.

[11] J.M. Nuñez, H. Bemelmans, J. Chromatogr. A 294 (1984) 361.

[12] G.P. Blanch, G. Reglero, M. Herraiz, J. Agric. Food Chem. 43 (1995) 1251.

[13] R. López, M. Aznar, J. Cacho, V. Ferreira, J. Chromatogr. A 966 (2002) 166.

[14] C. Cocito, G. Gaetano, C. Delfini, Food Chem. 52 (1995) 311.

[15] H. Lord, J. Pawliszyn, J. Chromatogr. A 885 (2000) 153.

[16] Z. Zhang, J. Pawliszyn, Anal. Chem. 65 (1993) 1843.

[17] E. Baltussen, P. Sandra, F. David, C. Cramers, J. Microcol. Sep. 11 (1999) 737.

[18] R.F. Alves, A.M.D. Nascimento, J.M.F. Nogueira, Anal. Chim. Acta 546 (2005) 11.

[19] J.S. Câmara, M.A. Alves, J.C. Marques, Anal. Chim. Acta 555 (2006) 191.

[20] E. Coelho, S.M. Rocha, I. Delgadillo, M.A. Coimbra, Anal. Chim. Acta 563 (2006) 204.

[21] S.M. Rocha, P. Coutinho, A. Barros, I. Delgadillo, M.A. Coimbra, J. Chromatogr. A 1114 (2006) 188.

[22] S. Rocha, V. Ramalheira, A. Barros, I. Delgadillo, M.A. Coimbra, J. Agric. Food Chem. 49 (2001) 5142.

[23] M.S. Burmeister, C.J. Drumond, E.A. Pfiesterer, D.W. Hysert, J. Am. Soc. Brew. Chem. 50 (1992) 53.

[24] O. Pinho, I.M.P.L.V.O. Ferreira, L.H.M.L.M. Santos, J. Chromatogr. A 1121 (2006) 145.

[25] J.C.R. Demyttenaere, C. Dagherb, P. Sandra, S. Kallithraka, R. Verhé, N. Kimpe, J. Chromatogr. A 985 (2003) 221.

[26] J.C.R. Demyttenaere, C. Dagherb, P. Sandra, S. Kallithraka, R. Verhé, N. Kimpe, J. Chromatogr. A 985 (2003) 233.

[27] J.S. Câmara, J.C. Marques, R. Perestrelo, F. Rodrigues, L. Oliveira, P. Andrade, M. Caldeira, J. Chromatogr. A 1150 (2007) 198.

[28] J.A. Pino, M.P. Martí, M. Mestres, J. Pérez, O. Busto, J. Guasch, J. Chromatogr. A 954 (2002) 51.

[29] J.A. Pino, R. Marbot, C. Vázquez, J. Agric. Food Chem. 49 (2001) 5883.

[30] J.A. Pino, R. Marbot, C. Vázquez, J. Agric. Food Chem. 50 (2002) 6023.

[31] F. Augusto, A.L.P. Valente, E.D. Tada, S.R. Rivellino, J. Chromatogr. A 873 (2000) 117.

[32] C. Deng, X. Zhang, N. Li, J. Chromatogr. B 808 (2004) 269.

[33] M. Ábalos, J.M. Bayona, J. Pawliszyn, J. Chromatogr. A 873 (2000) 107.

[34] T.A. Ternes, Water Res., 32 (12998) 3245.

[35] C. Deng, N. Li, X. Zhang, J. Chromatogr. B 808 (2004) 269.

[36] C. Deng, X. Zhang, N. Li, J. Chromatogr. B 813 (2004) 47. 